\title{
An analytical calculation of the Jacobian matrix for 3D friction contact model applied to turbine blade shroud contact
}

\author{
Mohammad Afzal $^{\mathrm{a}, *}$, Ines Lopez Arteaga ${ }^{\mathrm{a}, \mathrm{b}}$, Leif Kari ${ }^{\mathrm{a}}$ \\ ${ }^{a}$ KTH Royal Institute of Technology, The Marcus Wallenberg Laboratory for Sound and Vibration Research (MWL), SE 10044 \\ Stockholm, Sweden \\ ${ }^{b}$ Eindhoven University of Technology, Department of Mechanical Engineering, Section Dynamics and Control, P.O.Box 513, 5600 MB \\ Eindhoven, The Netherlands
}

\begin{abstract}
An analytical expression is formulated to compute the Jacobian matrix for 3D friction contact modelling that efficiently evaluates the matrix while computing the friction contact forces in the time domain by means of the alternate frequency time domain approach. The developed expression is successfully used for the calculation of the friction damping on a turbine blade with shroud contact interface having an arbitrary 3D relative displacement. The analytical expression drastically reduces the computation time of the Jacobian matrix with respect to the classical finite difference method, with many points at the contact interface. Therefore, it also significantly reduces the overall computation time for the solution of the equations of motion, since the formulation of the Jacobian matrix is the most time consuming step in solving the large set of nonlinear algebraic equations when a finite difference approach is employed. The equations of motion are formulated in the frequency domain using the multiharmonic balance method to accurately capture the nonlinear contact forces and displacements. Moreover, the equations of motion of the full turbine blade model are reduced to a single sector model by exploiting the concept of cyclic symmetry boundary condition for a periodic structure. Implementation of the developed scheme in solving the equations of motion is proved to be effective and significant reduction in time is achieved without loss of accuracy.
\end{abstract}

Keywords: Jacobian matrix; friction damping; shroud contact; cyclic symmetry; alternate frequency time domain method; multiharmonic balance method

\section{Introduction}

Rotating turbine blades are subjected to high static and dynamic loads during operation. The static loads are mainly caused by the centrifugal and thermal forces, whereas dynamic loads are induced by fluctuating gas forces. These stresses may lead to high cycle fatigue (HCF) [1-3]. The mechanical integrity of the turbine can be potentially ensured by keeping vibrations at a moderate level. To attenuate the high blade vibrations, dry friction damping [4] is widely used to dissipate the unwanted energy of the turbine blades. Dry friction damping is economical and potentially effective solution for wide range of mechanical systems and occurs at any contact interface that is in relative motion such as shroud coupling, underplatform dampers, lacing wires and in the turbine roots $[1,5-8]$, in the case of turbine bladed disk. The relative motion of the contact

${ }^{*}$ Corresponding author, Tel.: +46 700611121

Email addresses: mafzal@kth.se (Mohammad Afzal), inesla@kth.se (Ines Lopez Arteaga), leifkari@kth.se (Leif Kari) 
interfaces with preload generates friction forces and therefore provides additional stiffening and damping to the structure.

Earlier works [2,9-12] in the field of friction damping have demonstrated its effectiveness in attenuating turbine blade vibrations. These studies model contact interface as a macroslip and microslip element and consider 1D tangential relative motion, which may not be sophisticated enough to capture the arbitrary 3D motion of shroud contact. Menq et al. [13] successfully develop an analytical contact model for 1D tangential motion with inphase variable load and later propose an approximate method to capture the 2D planar motion with constant normal load [14]. A breakthrough in friction contact modelling is achieved by Yang et al. [7, 15-18], who establish the analytical criteria for 1-3D contact motion of friction interface in great detail. Petrov and Ewins [19] developed friction contact elements with an analytical expression of the tangent stiffness matrix in the frequency domain for a quasi-3D (two tangential directions are treated as uncoupled with variable normal load) friction contact model. These contact models can be referred to as a time-continuous scheme in which state (stick, slip or separation) transition times are resolved directly and an analytical expression is obtained between two states; however, the computation of state transition times often requires an iterative numerical procedure [20]. Although a time-continuous scheme leads to an accurate calculation of contact forces, a change in the contact model requires a new evaluation of the harmonic cofficients of the contact forces, which limits the flexibility of these contact models [21].

Sanliturk [22] develops a time-discrete algorithm for 2D coupled tangential motion with constant normal load, as an extension of Menq et al. [14]. The algorithm is not only restricted to Coulomb friction law. It can compute the friction forces based on theoretical models and measured friction force-displacement loading curves. The algorithm constitutes a basis for the further development of full-3D (two tangential directions are treated as coupled with variable normal load) friction contact models as presented by Shi Yajie [23] and $\mathrm{Gu}$ et al. [24, 25]. Moreover, Cameron and Griffin [26] develop an alternate frequency time domain method (AFT) which allows computation of the nonlinear friction forces in the time domain while solving the equations of motion (EQM) in the frequency domain. The EQM are formulated in the frequency domain using the classical harmonic balance method (HBM) $[15,27,28]$ and the recently more popular approach known as multiharmonic balance method (MHBM) [5, 8, 29], owing to their high computation efficiency compared to the time integration procedure. In conclusion, the AFT method combines the frequency domain EQM with friction forces computed in the time domain at discrete time steps, allowing to capture complex nonlinearities exhibited by the friction forces and other nonlinear behavior while keeping the computational advantage of frequency domain modelling. Accuracy of the time-discrete scheme is limited by the number of points chosen for the discretization; however, the approach is more flexible compared to the time-continuous scheme.

The EQM in the frequency domain constitute a set of nonlinear algebraic equations that are solved iteratively. The most common method employed in solving nonlinear algebraic equations is the Newton-Raphson method, which guarantees quadratic convergence in the neighborhood of the actual solution. An alternate to the Newton-Raphson method are quasi-Newton methods such as the secant and Broyden methods, which do not require the evaluation of the Jacobian at each iteration step; however, they are linear and superlinearly convergent in the neighborhood of the actual solution, respectively [30,31]. The most time consuming step in the Newton-Raphson method is the calculation of the Jacobian matrix, which is the partial derivatives of the harmonic coefficients of the friction forces with respect to the harmonic coefficient of the relative displacement. The classical finite difference approach for the evaluation of the Jacobian matrix is quite time consuming and it becomes cumbersome especially for the analysis of the mistuned blade assemblies and for the friction interfaces with multiple contact points. Moreover, the choice of the step size influences the value of the Jacobian matrix with the finite difference approach. Therefore, a fast and accurate method is required for this calculation. Petrov [19] and Borrajo et al. [32] have formulated the Jacobian matrix for a 
quasi-3D friction contact model and for the wedge damper case respectively; however, these formulations are obtained for the time-continuous scheme. Cardona [33] outlines a general procedure for computing the Jacobian matrix from the Fourier transform of the residual vector for a time-discrete formulation. Adopting the Cardona approach, Siewert [5] formulates an explicit analytical expression for the Jacobian matrix for a quasi-3D time-discrete friction contact model with variable normal load.

In this paper, the full-3D time-discrete friction contact model is reformulated and an analytical expression of the Jacobian matrix is derived. The developed scheme is successfully applied to a turbine blade with shroud contact and significant gain in computation efficiency is achieved while solving the EQM in the frequency domain. Furthermore, this formulation potentially makes the time-discrete scheme more attractive compared to the time-continuous scheme since analytical expression of the Jacobian matrix for a full-3D friction contact model in a time-continuous scheme is not known to the author. Moreover, this may lead to the industrial application of the full-3D friction contact model which is often restricted by the expensive calculation of the Jacobian matrix while using a classical finite difference approach.

\section{Equation of motion of a single sector in complex form}

The dynamic response of a rotationally periodic structure with identical sectors such as a turbine blade can be analyzed using a single sector model by applying complex cyclic constraints at the disk interface [34]. Before applying the complex cyclic constraints, the EQM of the $k^{\text {th }}$ sector can be written as

$$
\mathbf{M}^{(k)} \ddot{\mathbf{q}}(t)+\mathbf{C}^{(k)} \dot{\mathbf{q}}(t)+\mathbf{K}^{(k)} \mathbf{q}(t)={ }^{(k)} \mathbf{f}_{\mathrm{ext}}(t)-{ }^{(k)} \mathbf{f}_{\mathrm{nl}}\left(\mathbf{q}_{\mathrm{nl}}(t), \dot{\mathbf{q}}_{\mathrm{nl}}(t), t\right),
$$

where $\mathbf{M}, \mathbf{K}$ and $\mathbf{C}$ are the mass, stiffness and viscous damping matrices of a single sector, ${ }^{(k)} \mathbf{q}(t)$ represents

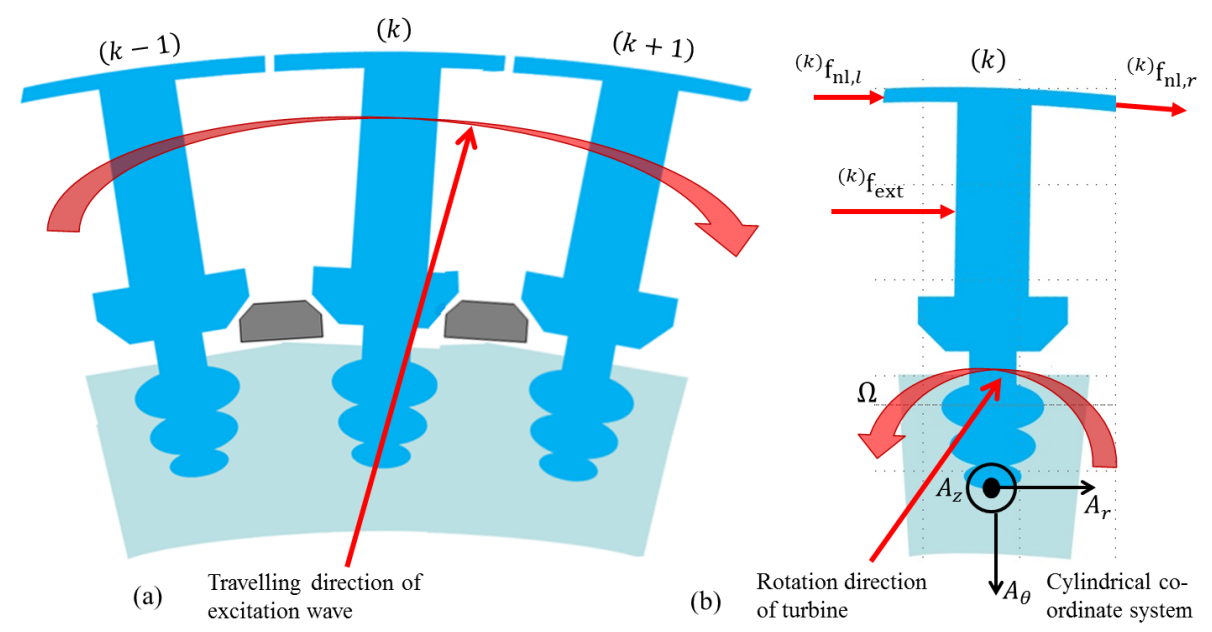

Figure 1: (a) Travelling excitation wave (b) Single sector model in cylindrical coordinate system

the cyclic displacement vector of the $k^{\text {th }}$ sector, which is equivalent to the displacements in the cylindrical coordinate system for a sector model and ${ }^{(k)} \mathbf{f}_{\text {ext }}(t)$ is a travelling wave excitation [8, 21, 35]. A dot above $\mathbf{q}(t)$ indicates derivative with respect to time, $t$. Due to the spatial periodicity of the excitation wave, it is expressed as a Fourier series truncated after $n_{h}$ harmonics. Note that truncation leads to a loss of accuracy and therefore a sufficient number of harmonics must be chosen to approximate the dynamic response of the 
structure. Transformation of excitation wave into the cyclic rotating frame of reference fixed to the rotor results into a periodic excitation force on the $k^{\text {th }}$ blade, which reads

$$
{ }^{(k)} \mathbf{f}_{\mathrm{ext}}(t) \approx \operatorname{Re}\left\{\sum_{n=0}^{n_{h}}{ }^{(k)} \hat{\mathbf{f}}_{\mathrm{ext}} \mathrm{e}^{\mathrm{i} n \tau}\right\}=\operatorname{Re}\left\{\sum_{n=0}^{n_{h}}{ }^{(1)} \hat{\mathbf{f}}_{\mathrm{ext}} \mathrm{e}^{\mathrm{i} n\left(\tau-(k-1) \phi_{m}\right)}\right\},
$$

where $\mathrm{i}$ is the imaginary unit and $k=1,2, \ldots, n_{b}$ with $n_{b}$ the number of identical sectors. The phase shift is defined as $\phi_{m}=m \phi$ with $\phi=2 \pi / n_{b}$ the interblade phase angle, $m$ the spatial periodicity and the nondimensional time is defined as $\tau=m \Omega t$ with $\Omega$ the rotation speed. It can be seen from Eq. (2) that the excitation for the $k^{\text {th }}$ sector can be derived from the $1^{\text {st }}$ sector due to the spatial periodicity of the excitation pattern. The vector ${ }^{(k)} \mathbf{f}_{\mathrm{nl}}\left(\mathbf{q}_{\mathrm{nl}}(t), \dot{\mathbf{q}}_{\mathrm{nl}}(t), t\right)$ is the nonlinear contact force caused by friction and the relative displacement of the contact interface nodes. The displacements of the contact interface nodes are referred to as nonlinear displacements in this paper and denoted $\mathbf{q}_{\mathrm{nl}}(t)$.

In the case of periodic excitation, it is usually desirable to find the steady-state periodic response of the structure. Therefore, the displacement vector ${ }^{(k)} \mathbf{q}(t)$ and nonlinear interaction force ${ }^{(k)} \mathbf{f}_{\mathrm{nl}}\left(\mathbf{q}_{\mathrm{nl}}, \dot{\mathbf{q}}_{\mathrm{n} l}, t\right)$ are truncated after $n_{h}$ harmonics as

$$
{ }^{(k)} \mathbf{q}(t)=\operatorname{Re}\left\{\sum_{n=0}^{n_{h}}{ }^{(k)} \hat{\mathbf{q}}_{n} \mathrm{e}^{\mathrm{i} n \tau}\right\}
$$

and

$$
{ }^{(k)} \mathbf{f}_{\mathrm{nl}}\left(\mathbf{q}_{\mathrm{nl}}, \dot{\mathbf{q}}_{\mathrm{nl}}, t\right)=\operatorname{Re}\left\{\sum_{n=0}^{n_{h}}{ }^{(k)} \hat{\mathbf{f}}_{\mathrm{n} 1, n} \mathrm{e}^{\mathrm{i} n \tau}\right\},
$$

where ${ }^{(k)} \hat{\mathbf{q}}_{n}$ is the $n^{\text {th }}$ temporal harmonic coefficient of the displacement vector of the $k^{\text {th }}$ blade, with the length of each vector equal to the number of degree of freedoms (DOFs) in a single sector. The vector ${ }^{(k)} \hat{\mathbf{f}}_{\mathrm{nl}, n}$ is the $n^{\text {th }}$ temporal harmonic coefficient of the nonlinear contact forces, which only depend on the nonlinear DOFs at the shroud contact.

Inserting Eqs. (2)-(4), into Eq. (1) and by applying Fourier-Galerkin's method [36], the EQM in the frequency domain are formulated as

$$
\hat{\mathbf{D}}(m, n, \Omega) \hat{\mathbf{q}}+\hat{\mathbf{f}}_{\mathrm{nl}}\left(\hat{\mathbf{q}}_{\mathrm{nl}}, m, n, \Omega\right)-\hat{\mathbf{f}}_{\mathrm{ext}} \approx \mathbf{0} .
$$

Since EQM are formulated for a single sector, the left superscript $(k)$ is omitted for brevity here and in the following. Here, the vector $\hat{\mathbf{q}}$ consists of all the DOFs $\left(n_{s}\right)$ and $\hat{\mathbf{q}}_{\mathrm{nl}}$ contains only the nonlinear DOFs $\left(n_{\mathrm{nl}}\right)$ of a single sector. Since each DOF is described by $\left(n_{h}+1\right)$ harmonics, $\hat{\mathbf{q}}$ and $\hat{\mathbf{q}}_{\mathrm{nl}}$ have length $n_{s}\left(n_{h}+1\right)$ and $n_{\mathrm{nl}}\left(n_{h}+1\right)$, respectively, and are assembled as

$$
\hat{\mathbf{q}}=\left[\begin{array}{c}
\hat{\mathbf{q}}_{0} \\
\hat{\mathbf{q}}_{1} \\
\vdots \\
\hat{\mathbf{q}}_{n_{h}}
\end{array}\right] \text { and } \hat{\mathbf{q}}_{\mathrm{nl}}=\left[\begin{array}{c}
\hat{\mathbf{q}}_{\mathrm{nl}, 0} \\
\hat{\mathbf{q}}_{\mathrm{nl}, 1} \\
\vdots \\
\hat{\mathbf{q}}_{\mathrm{nl}, n_{h}}
\end{array}\right] .
$$

The vector $\hat{\mathbf{q}}_{n}$ contains the harmonic coefficients of the $n^{\text {th }}$ harmonic as defined in Eq. (3) and $\hat{\mathbf{q}}_{\mathrm{nl}, n}$ are the subset of $\hat{\mathbf{q}}_{n}$ containing the nonlinear DOFs of the single sector. Note that the nonlinear friction forces at the shroud contact also depend on the nonlinear DOFs of the $(k-1)^{\text {th }}$ and $(k+1)^{\text {th }}$ sectors; however, displacements of these nonlinear DOFs are obtained using $k^{\text {th }}$ sector as explained below. Harmonics of the 
travelling wave excitation are assembled in the vector $\hat{\mathbf{f}}_{\text {ext }}$. The dynamic stiffness matrix $\hat{\mathbf{D}}$ is a diagonal matrix and reads as

$$
\hat{\mathbf{D}}=\operatorname{diag}\left(\hat{\mathbf{D}}_{0}, \hat{\mathbf{D}}_{1} \ldots \hat{\mathbf{D}}_{n_{h}}\right),
$$

where each submatrix takes the form

$$
\hat{\mathbf{D}}_{n}=\mathbf{K}-(n m \Omega)^{2} \mathbf{M}+\mathrm{i} n m \Omega \mathbf{C},
$$

with $n=0,1, \ldots, n_{h}$ and diag is diagonal operator. Here, $n=0$ represents the static equilibrium equation and $n=1,2, \ldots, n_{h}$ represents the dynamic equilibrium equations. Note that it is important to solve the coupled static and dynamic equations of Eq. (5) in a single iteration loop since the static component of the nonlinear force varies at each iteration step and therefore plays an important role in the effective friction damping. Solving the static and dynamic equilibrium equations separately may lead to convergence problem as well.

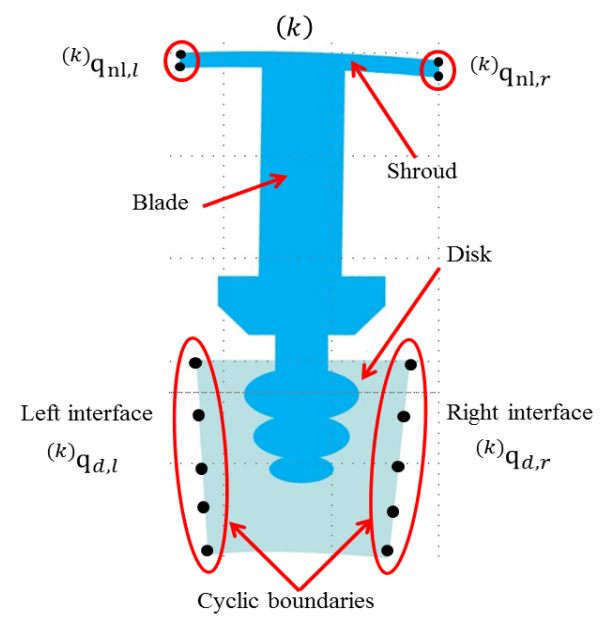

Figure 2: Nodes at the disk interface and shroud contact

To compute the nonlinear response of a full turbine bladed disk using a single sector model, complex cyclic constraints are required at two locations: cyclic boundaries and shroud contact. The cyclic constraints at the cyclic boundaries allow for the calculation of the nodal diameter map [34] while the constrains at the shroud contact facilitate the calculation of the nonlinear contact force using a single sector instead of the full bladed disk, see Fig. 1. A detailed procedure for implementation of the complex cyclic constraints at the cyclic boundaries can be found in Refs. [5,35]. By applying the cyclic constrains, displacements of the right interface nodes are obtained from the displacements of the left interface nodes (Fig. 2) and therefore the right cyclic boundary nodes are eliminated from the EQM and the EQM are reduced to

$$
\tilde{\mathbf{D}}(m, n, \Omega) \tilde{\mathbf{q}}+\tilde{\mathbf{f}}_{\mathrm{nl}}\left(\hat{\mathbf{q}}_{\mathrm{nl}}, m, n, \Omega\right)-\tilde{\mathbf{f}}_{\mathrm{ext}}=\mathbf{0},
$$

where $\tilde{\mathbf{q}}$ consists of the harmonic coefficients of the displacements vector eliminating the right interface DOFs from $\hat{\mathbf{q}}$. The matrix $\tilde{\mathbf{D}}, \tilde{\mathbf{f}}_{\mathrm{nl}}$ and $\tilde{\mathbf{f}}_{\text {ext }}$ are the dynamic stiffness matrix, the nonlinear force vector and the excitation force vector after the implementation of the cyclic constraints. As a result of the transformation, $\tilde{\mathbf{D}}$ becomes Hermitian and therefore the mass and stiffness matrix of the cyclic single sector turn into a Hermitian matrix, which depends on phase angle $\phi_{m}$ and temporal harmonic, $n$. Thus for the different values 
of $\phi_{m}$ and $n$ the eigenvalue problem of the cyclic sector model yields to the eigenvectors and mode shapes of the corresponding nodal diameter. The corresponding nodal diameter is obtained from $m, n$ and $n_{b}$, see Ref. [21]. Note that the eigenvectors of the hermitian matrix are complex and represent a rotating waveform instead of a standing wave [34].

The final step in the reduction of the EQM to a single sector model is the implementation of cyclic constraints at the shroud contact, which results in the relative displacements at the right and left shroud interface of the $k^{\text {th }}$ blade

$$
\mathbf{w}_{\mathrm{nl}, r}(t)={ }^{(k)} \mathbf{q}_{\mathrm{nl}, r}(t)-{ }^{(k+1)} \mathbf{q}_{\mathrm{nl}, l}(t)=\operatorname{Re}\left\{\sum_{n=0}^{n_{h}}\left({ }^{(k)} \hat{\mathbf{q}}_{\mathrm{nl}, n}-e^{-\mathrm{i} n \phi_{m}(k)} \hat{\mathbf{q}}_{\mathrm{nll}, n}\right) e^{\mathrm{i} n \tau}\right\}
$$

and

$$
\mathbf{W}_{\mathrm{nl}, l}(t)={ }^{(k)} \mathbf{q}_{\mathrm{nl}, l}(t)-{ }^{(k-1)} \mathbf{q}_{\mathrm{nl}, r}(t)=\operatorname{Re}\left\{\sum_{n=0}^{n_{h}}\left({ }^{(k)} \hat{\mathbf{q}}_{\mathrm{nll}, n}-e^{\mathrm{i} n \phi_{m}(k)} \hat{\mathbf{q}}_{\mathrm{nlr}, n}\right) e^{\mathrm{i} n \tau}\right\},
$$

where $\mathbf{w}_{\mathrm{nl}, r}(t)$ and $\mathbf{w}_{\mathrm{nl}, l}(t)$ are the relative displacement vector at the right and left shroud interfaces for all the contacting nodes. Note that ${ }^{(k)} \mathbf{q}_{\mathrm{nl}, r}$ and ${ }^{(k+1)} \mathbf{q}_{\mathrm{nl}, l}$ are the coinciding nodes at the right interface of the $k^{\text {th }}$ sector. The vectors $\hat{\mathbf{q}}_{\mathrm{nl}, n}$ and $\hat{\mathbf{q}}_{\mathrm{nll}, n}$ are the $n^{\text {th }}$ harmonic coefficient of the displacements of nonlinear DOFs at the right and left shroud contact respectively. Left superscript $(k-1, k$ and $k+1)$ are used to distinguish between the different sectors. The nonlinear contact forces can be directly calculated using Eqs. (10)-(11) and the 3D contact model described in the next section. Nevertheless, due to the assumed cyclic symmetry and because of Newton's third law, nonlinear contact forces on the right shroud contact can be directly computed from the contact forces on the left shroud contact in the cyclic coordinate system and vice versa, reads as

$$
\mathbf{f}_{\mathrm{nl}, r}(t)=\operatorname{Re}\left\{\sum_{n=0}^{n_{h}} \hat{\mathbf{f}}_{\mathrm{nlr}, n} e^{\mathrm{i} n \tau}\right\}=-\operatorname{Re}\left\{\sum_{n=0}^{n_{h}} e^{-\mathrm{i} n \phi_{m}} \hat{\mathbf{f}}_{\mathrm{nll}, n} e^{\mathrm{i} n \tau}\right\},
$$

where $\mathbf{f}_{\mathrm{nl}, r}(t)$ represents the nonlinear contact forces at the right shroud contact and $\hat{\mathbf{f}}_{\mathrm{nlr}, n}$ and $\hat{\mathbf{f}}_{\mathrm{nll}, n}$ are the $n^{\text {th }}$ harmonic coefficient of the right and left shroud nonlinear contact forces, respectively. Note that the formulation described in this section is defined in a cyclic coordinate system while the contact modelling described in the next section is defined in the local Cartesian coordinate system of the shroud. Therefore, the relative displacements in Eqs. (10)-(11) are to be converted to the shroud local coordinate system before using the contact model. Similarly, the nonlinear contact forces are computed in the shroud local coordinate system and are to be converted back to the cyclic coordinate system before substituting in the EQM, Eq. (9).

\section{Contact model}

A contact model is described by the contact laws between the contact interfaces that determine the contact forces. A linear-elastic unilateral law is considered in the normal direction and a regularized form of Coulomb's law in the frame work of elasto-plastic constitutive relations $[37,38]$ is used in the tangential plane to characterize the contact forces between the interfaces. An elastic formulation of the contact laws is used to avoid the constraint optimization problem arises due to the non-smooth rigid contact laws [39] and it is well adapted to the solution method used in this paper. Note that a regularized formulation does not remove the non-smoothness of the contact law completely and may lead to numerical difficulties with high contact stiffness. A smoothing variant of the friction contact law and an algorithm based on a quadratic formulation of the friction contact law for quasi-static finite displacement problems are presented in Refs. [40] 
and [41], respectively. These contact models are likely to reduce the numerical problems during solution, but to the authors' knowledge these models have not been used in the context of dynamic friction contact problems, where deformations are small relative to the static equilibrium position. Nevertheless, due to the frequency domain approach (MHBM) used in the analysis, the contact forces are smoothen by truncating the Fourier series after few harmonics and global vibration behavior are often predicted with sufficient accuracy using the MHBM approach, as compared with time integration of the non-smooth model [42].

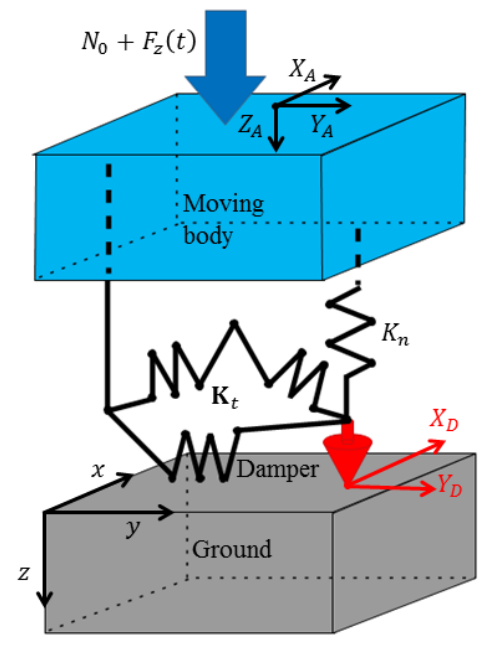

Figure 3: The 3D friction contact model

A time-discrete variant of full-3D friction contact model is reformulated (Fig. 3) in this section, and an efficient procedure to formulate its Jacobian matrix is described. Although the proposed contact model in this paper is similar to the model proposed in Refs. [23, 24] and an extension of the 2D contact model developed in Ref. [22], the equations are reformulated in order to compute the Jacobian matrix in parallel with the contact forces. Moreover, the trajectory of motion at the contact is arbitrary and not limited to circular and elliptical trajectories. The full-3D relative motion of the shroud interface comprises the coupled 2D tangential motion in the plane of the shroud and the normal motion perpendicular to the tangential plane. The tangential motion induces stick-slip phenomena and therefore it provides addition stiffness and damping to the structure whereas the normal motion alters the normal contact load and provides additional stiffness in the normal contact direction. In an elastic formulation, friction contact forces can be fully characterized by the relative motion of the contact interface $(A)$ and the motion of the damper $(D)$, attached to the moving body $(A)$ by two linear springs $\left(\mathbf{K}_{t}\right.$ and $\left.K_{n}\right)$, that represent the shear and normal contact stiffness respectively, see Fig. 3 and Refs. [23, 24]. The matrix $\mathbf{K}_{t}$ is a $(2 \times 2)$ matrix that reads as

$$
\mathbf{K}_{t}=\left[\begin{array}{ll}
K_{x x} & K_{x y} \\
K_{y x} & K_{y y}
\end{array}\right] .
$$

In the case of an isotropic material which is a reasonable assumption for the shroud contact $\mathbf{K}_{t}$ can be simplified as $K_{x x}=K_{y y}=K_{t}$ and $K_{x y}=K_{y x}=0$, moreover the formulation is valid for an anisotropic material as well. Note that the relative motion of $(A)$ is known from the EQM (Eq. (9)) while the motion of the damper $(D)$ should be computed numerically using the full-3D friction contact model described below. 


\subsection{The time-discrete full-3D friction contact model}

In Fig. 3, $\left(X_{A}, Y_{A}, Z_{A}\right)$ refers to the relative displacement of $(A)$ in the local coordinate system of the shroud and $\left(X_{D}, Y_{D}, Z_{D}\right)$ is the displacement of the damper $(D)$ in the same coordinate system. The static component of the normal load $N_{0}$ is induced by centrifugal forces and $F_{z}(t)$ is the variable component of normal load defined as $K_{n} Z_{A}(t)$. The tangential frictional force is governed by the elasto-plastic Coulomb's law and the proposed model includes the possibility to model the initial gap as $G=-N_{0} / K_{n}$. In this formulation, the damper always moves in the tangential plane and therefore the normal component of the damper displacement $Z_{D}=0$ for all the contact states (stick, slip or separation). However, the normal component of the relative displacement $Z_{A} \neq 0$, leading to load variation in the normal direction, see Eq. (15). To trace the tangential motion $\left(X_{D}, Y_{D}\right)$ of the damper, the motion trajectory of $(A)$ is discretized in $N$ points for one cycle of the periodic motion. Thus, $X_{A}(l), Y_{A}(l), Z_{A}(l)[l=1,2, \ldots ., N]$ represent the relative displacement of $(A)$ at $l^{\text {th }}$ discrete point. Based on these descriptions and assuming that the direction of the friction force is coincident with the damper velocity,

$$
\frac{\mathbf{F}_{t}}{\left\|\mathbf{F}_{t}\right\|}=\frac{\mathbf{V}_{D}}{\left\|\mathbf{V}_{D}\right\|},
$$

where the magnitude of friction force $\left\|\mathbf{F}_{t}\right\|=\sqrt{F_{t x}^{2}(l)+F_{t y}^{2}(l)}$ and the damper velocity vector $\mathbf{V}_{D}=\left[\dot{X}_{D}(l), \dot{Y}_{D}(l)\right]^{\mathrm{T}}$ in the tangential plane. The total variable normal load $F_{n z}(l)$ and the friction force $\mathbf{F}_{t}(l)=\left[F_{t x}(l), F_{t y}(l)\right]^{\mathrm{T}}$ at the $l^{\text {th }}$ discrete point are given by

$$
\begin{gathered}
F_{n z}(l)= \begin{cases}0 & \text { if } N_{0}+K_{n} Z_{A}(l) \leq 0 \text { Separation } \\
N_{0}+K_{n} Z_{A}(l) & \text { if } N_{0}+K_{n} Z_{A}(l)>0 \text { Contact },\end{cases} \\
F_{t x}(l)= \begin{cases}0 & \text { if } F_{n z}(l)=0 \text { Separation } \\
K_{t}\left[X_{A}(l)-X_{D}(l)\right] & \text { if }\left|\mathbf{F}_{t}(l)\right|<\mu F_{n z}(l) \text { Stick } \\
\mu F_{n z}(l) \frac{\dot{X}_{D}(l)}{\sqrt{\dot{X}_{D}^{2}(l)+\dot{Y}_{D}^{2}(l)}} & \text { if }\left|\mathbf{F}_{t}(l)\right|=\mu F_{n z}(l) \text { Slip }\end{cases}
\end{gathered}
$$

and

$$
F_{t y}(l)=\left\{\begin{array}{ll}
0 & \text { if } F_{n z}(l)=0 \text { Separation } \\
K_{t}\left[Y_{A}(l)-Y_{D}(l)\right] & \text { if }\left|\mathbf{F}_{t}(l)\right|<\mu F_{n z}(l) \text { Stick } \\
\mu F_{n z}(l) \frac{\dot{Y}_{D}(l)}{\sqrt{\dot{X}_{D}^{2}(l)+\dot{Y}_{D}^{2}(l)}} & \text { if }\left|\mathbf{F}_{t}(l)\right|=\mu F_{n z}(l) \text { Slip }
\end{array},\right.
$$

where $\mu$ is the friction coefficient and $\mathrm{T}$ is transpose. The only unknowns in the above equations are the motion trajectory of $(D)$ and its derivatives. To trace the stable trajectory of $(D)$, iteration over several periods of $(A)$ is required, until the error between the $(M-1)^{\text {th }}$ and $M^{\text {th }}$ period is negligible. Often the value of $M$ is between 3 and 5. To start the iteration loop, the initial value of $(D)$ is assumed to be zero $\left(X_{D}=0, Y_{D}=0\right)$ and the friction forces $\left[F_{t x}(l), F_{t y}(l)\right]^{\mathrm{T}}$ are calculated using Eqs. (16)-(17) under the stick state assumption. If the resultant friction force $\left\|\mathbf{F}_{t}(l)\right\| \leq \mu F_{n z}(l)$, then $(D)$ remains stationary, i.e, $\left[X_{D}(l)=X_{D}(l-1)\right.$ and $\left.Y_{D}(l)=Y_{D}(l-1), l=1,2,3 \ldots\right]$. On the other hand, if $\left\|\mathbf{F}_{t}(l)\right\|>\mu F_{n z}(l)$ then $(D)$ moves such that the frictional force becomes equal to $\mu F_{n z}(l)$. Moreover, $(D)$ moves along the line joining $D(l-1)$ and $A(l)$ as seen in Fig. 4 and therefore the friction force also has the same direction. This leads to 
the following expression for the direction cosine in the $x$-direction for the slip state

$$
\frac{\dot{X}_{D}(l)}{\sqrt{\dot{X}_{D}^{2}(l)+\dot{Y}_{D}^{2}(l)}}=\frac{X_{A}(l)-X_{D}(l-1)}{d(l)} .
$$

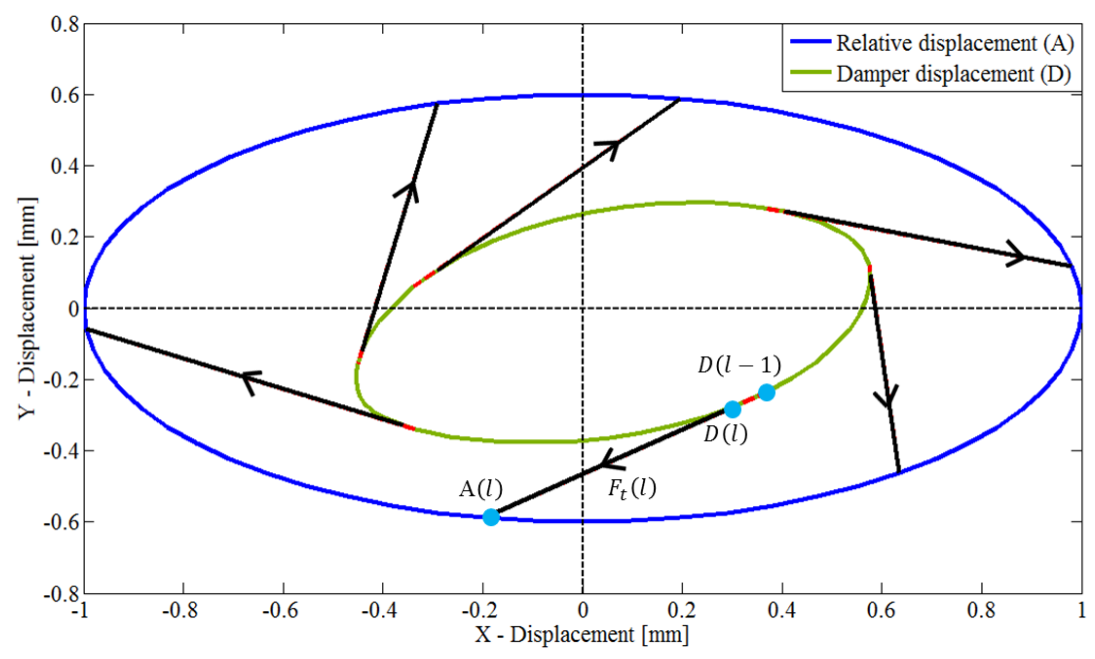

Figure 4: Tangential motion of the damper and the friction force

Subsequently, the damper displacement $(D)$ in $x$-direction is evaluated as

$$
X_{D}(l)= \begin{cases}X_{A}(l) & \text { Separation } \\ X_{D}(l-1) & \text { Stick } \\ X_{A}(l)-\frac{F_{t x}(l)}{K_{t}} & \text { Slip }\end{cases}
$$

where $d(l)=\sqrt{\left[X_{A}(l)-X_{D}(l-1)\right]^{2}+\left[Y_{A}(l)-Y_{D}(l-1)\right]^{2}}$. Analogous expressions for the direction cosine and the damper displacement in $y$-direction can be obtained. Note that in case of separation, the displacement of $(D)$ follows $(A)$. Substituting Eqs. (18)-(19) into Eqs. (16)-(17) results in the following expressions for the friction forces of an arbitrary planar motion

$$
F_{t x}(l)= \begin{cases}0 & \text { Separation } \\ K_{t}\left[X_{A}(l)-X_{D}(l-1)\right]=K_{t}\left[X_{A}(l)-X_{A}(l-1)\right]+F_{t x}(l-1) & \text { Stick } \\ \mu F_{n z}(l) \frac{\left[X_{A}(l)-X_{D}(l-1)\right]}{d(l)} & \text { Slip }\end{cases}
$$

and

$$
F_{t y}(l)=\left\{\begin{array}{ll}
0 & \text { Separation } \\
K_{t}\left[Y_{A}(l)-Y_{D}(l-1)\right]=K_{t}\left[Y_{A}(l)-Y_{A}(l-1)\right]+F_{t y}(l-1) & \text { Stick } \\
\mu F_{n z}(l) \frac{\left[Y_{A}(l)-Y_{D}(l-1)\right]}{d(l)} & \text { Slip }
\end{array} .\right.
$$


Using Eqs. (15) and (19)-(21), the time history of $(D)$ and the nonlinear contact forces can be computed for a given periodic displacement of $(A)$. Since the EQM (9) for the steady-state response calculation are formulated in the frequency domain using the MHBM while the nonlinear contact forces are in the time domain, the AFT method developed in [26] is employed in order to switch between frequency and time domain, see Fig. 5. In the AFT procedure, first Fourier coefficients of the relative displacements $\left(\hat{X}_{A, n}, \hat{Y}_{A, n}\right.$ and $\left.\hat{Z}_{A, n}\right)$ of $(A)$ are converted into the time domain using inverse FFT. This results in the time history of the relative displacement at the $l^{\text {th }}$ step

$$
X_{A}(l)=\sum_{n=0}^{N-1} \hat{X}_{A, n} e^{\mathrm{i} n l 2 \pi / N} \text { for } l=0,1, \ldots, N-1,
$$

where $N$ denotes the size of the FFT and number of discrete points in one periodic cycle. Since the required

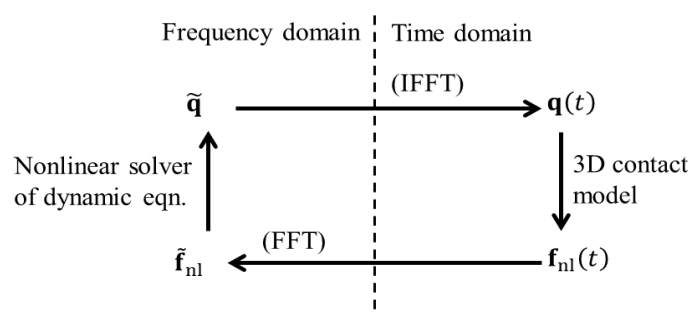

Figure 5: AFT flow diagram

number of Fourier coefficients in the MHBM formulation are fewer than $N$, i.e. $\left(n_{h}<<N\right)$, the intermediate Fourier coefficients $\left(n_{h}<n<N-n_{h}-1\right)$ are set to zero in the Eq. (22). Similarly, expressions for $Y_{A}(l)$ and $Z_{A}(l)$ are obtained. Subsequently, the damper motion and hence the nonlinear contact forces in the time domain are determined as described previously in this section. Finally, the corresponding Fourier coefficients of the normal contact force are

$$
\hat{F}_{n z, n}=\frac{1}{N} \sum_{l=0}^{N-1} F_{n z}(l) e^{-\mathrm{i} n l 2 \pi / N} \text { for } n=0,1, \ldots, N-1,
$$

using FFT and an analogous expression for the Fourier coefficients of the tangential contact forces is derived to solve the nonlinear EQM. This completes a full cycle of the AFT flow diagram that is performed at each iteration step, see Fig. 5. The advantage of the AFT method is that it makes extensive use of the FFT algorithm in order to reduce the computational time. Furthermore, since the nonlinear forces are evaluated in the time domain, this facilitates the straightforward computation of the complex nonlinearities such as stick-slip behavior associated with the friction forces. Consequently, the accuracy of the AFT method is only restricted by the number of temporal harmonics considered in the MHBM formulation and the size of FFT $(N)$.

\subsection{The Jacobian matrix}

The cyclic sector of the turbine bladed disk contains a large number of DOFs even though it has been reduced to a single sector, see Eq. (9). An iterative solution with such a large number of DOFs is cost-intensive and computationally inefficient as well. However, since the nonlinear contact force only depends on the relative motion of the nonlinear DOFs, the size of the EQM can be significantly reduced by keeping only nonlinear 
DOFs in the system. This is achieved by applying the dynamic compliance matrix method that builds the receptance matrix based on the normal complex mode shapes of the structure as presented in Ref. [21]. Applying the dynamic compliance matrix method on the EQM (Eq. (9)), and partitioning the linear and nonlinear DOFs, the EQM can be rewritten as

$$
\left[\begin{array}{c}
\tilde{\mathbf{q}}_{\text {lin }} \\
\tilde{\mathbf{q}}_{\mathrm{nl}}
\end{array}\right]=\left[\begin{array}{cc}
\tilde{\mathbf{R}}_{\text {lin,lin }}(m, n, \Omega) & \tilde{\mathbf{R}}_{\mathrm{lin}, \mathrm{nl}}(m, n, \Omega) \\
\tilde{\mathbf{R}}_{\mathrm{nl}, \text { lin }}(m, n, \Omega) & \tilde{\mathbf{R}}_{\mathrm{nl}, \mathrm{nl}}(m, n, \Omega)
\end{array}\right]\left\{\left[\begin{array}{c}
\tilde{\mathbf{f}}_{\mathrm{ext}, \mathrm{lin}} \\
\tilde{\mathbf{f}}_{\mathrm{ext}, \mathrm{nl}}
\end{array}\right]-\left[\begin{array}{c}
\mathbf{0} \\
\tilde{\mathbf{f}}_{\mathrm{nl}}\left(\tilde{\mathbf{q}}_{\mathrm{nl}}, m, n, \Omega\right)
\end{array}\right]\right\},
$$

leading to

$$
\tilde{\mathbf{q}}_{\mathrm{nl}}-\tilde{\mathbf{q}}_{\mathrm{nl}, 0}+\tilde{\mathbf{R}}_{\mathrm{nl}, \mathrm{nl}}(m, n, \Omega) \tilde{\mathbf{f}}_{\mathrm{nl}}\left(\tilde{\mathbf{q}}_{\mathrm{nl}}, m, n, \Omega\right)=0,
$$

where $\tilde{\mathbf{q}}_{\mathrm{nl}, 0}=\tilde{\mathbf{R}}_{\mathrm{nl}, \text { lin }}(m, n, \Omega) \hat{\mathbf{f}}_{\mathrm{ext}, \text { lin }}+\tilde{\mathbf{R}}_{\mathrm{nl}, \mathrm{nl}}(m, n, \Omega) \hat{\mathbf{f}}_{\mathrm{ext}, \mathrm{nl}}$ represents the linear response of the nonlinear DOFs in the absence of the friction contact. The matrix $\tilde{\mathbf{R}}_{\mathrm{nl}, \mathrm{nl}}(m, n, \Omega)$ is the nonlinear part of the dynamic compliance matrix (FRF matrix), which is the inverse of $\tilde{\mathbf{D}}_{\mathrm{nl}, \mathrm{nl}}$, that is a part of the full dynamic stiffness matrix $\tilde{\mathbf{D}}(m, n, \Omega)$, see Eq. (9). To avoid the inefficient evaluation of the inverse of a matrix, the FRF is computed based on modal superposition as explained in Ref. [21].

The Newton-Raphson method is applied to solve the reduced Eq. (25). An iterative step for Eq. (25) can be expressed as

$$
\tilde{\mathbf{q}}_{\mathrm{nl}}^{(p+1)}=\tilde{\mathbf{q}}_{\mathrm{nl}}^{(p)}-\left\{\frac{\partial \mathbf{e}\left(\tilde{\mathbf{q}}_{\mathrm{nl}}^{(p)}\right)}{\partial \tilde{\mathbf{q}}_{\mathrm{nl}}^{(p)}}\right\}^{-1} \mathbf{e}\left(\tilde{\mathbf{q}}_{\mathrm{nl}}^{(p)}\right)
$$

where $\tilde{\mathbf{q}}_{\mathrm{nl}}^{(p)}$ and $\mathbf{e}\left(\tilde{\mathbf{q}}_{\mathrm{nl}}^{(p)}\right)$ represent the nonlinear displacement vector and the residual vector at the $p^{\text {th }}$ iteration step, respectively. The residual vector at the $p^{\text {th }}$ step is given by

$$
\mathbf{e}\left(\tilde{\mathbf{q}}_{\mathrm{nl}}^{(p)}\right)=\tilde{\mathbf{q}}_{\mathrm{nl}}^{(p)}-\tilde{\mathbf{q}}_{\mathrm{nl}, 0}+\tilde{\mathbf{R}}_{\mathrm{nl}, \mathrm{nl}}(m, n, \Omega) \tilde{\mathbf{f}}_{\mathrm{nl}}^{(p)}\left(\tilde{\mathbf{q}}_{\mathrm{nl}}^{(p)}, m, n, \Omega\right)
$$

and the Jacobian matrix at the $p^{\text {th }}$ iteration step reads as

$$
\tilde{\mathbf{J}}^{(p)}=\left\{\frac{\partial \mathbf{e}\left(\tilde{\mathbf{q}}_{\mathrm{nl}}^{(p)}\right)}{\partial \tilde{\mathbf{q}}_{\mathrm{nl}}^{(p)}}\right\}=\mathbf{I}_{n_{\mathrm{nl}}\left(n_{h}+1\right)}+\tilde{\mathbf{R}}_{\mathrm{nl}, \mathrm{nl}}(m, n, \Omega)\left\{\frac{\partial \tilde{\mathbf{f}}_{\mathrm{nl}}^{(p)}}{\partial \tilde{\mathbf{q}}_{\mathrm{nl}}^{(p)}}\right\}
$$

Note that the above operations are performed using complex arithmetic and that Eq. (25) is not an analytical function of the complex vector $\tilde{\mathbf{q}}_{\mathrm{nl}}$, which means that the Jacobian matrix in Eq. (28) is undefined [35]. Therefore, Eqs. (26)-(28) can not be used directly in complex domain and Eq. (25) must be transformed into real arithmetic in order to apply the Newton-Raphson method. These transformations can be accomplished using the Kronecker product defined in Appendix A. Applying the real arithmetic transformation, the Jacobian matrix reads as

$$
\tilde{\mathbf{J}}_{r}^{(p)}=\mathbf{I}_{2 n_{\mathrm{nl}}\left(2 n_{h}+2\right)}+\operatorname{Re}\left\{\tilde{\mathbf{R}}_{\mathrm{nl}, \mathrm{nl}}(m, n, \Omega) \otimes\left[\begin{array}{cc}
1 & \mathrm{i} \\
-\mathrm{i} & 1
\end{array}\right]\right\}\left\{\mathbf{T}_{s c}\right\} \tilde{\mathbf{J}}_{s}^{(p)},
$$

where $\tilde{\mathbf{J}}_{r}^{(p)}$ is the Jacobian matrix in real arithmetic form at the $p^{\text {th }}$ iteration step and $\mathbf{T}_{s c}$ represents the coordinate transformation matrix from the local shroud coordinate system to the global cyclic coordinate system. The matrix $\tilde{\mathbf{J}}_{s}$ contains the partial derivative of the Fourier coefficients of the nonlinear contact forces with respect to the Fourier coefficients of the relative displacements in the local shroud coordinate system. Expression of the matrix $\tilde{\mathbf{J}}_{s}$ for a single node-to-node contact with 3 DOFs $(x, y, z)$, is given in Eq. 
(30).

$$
\tilde{\mathbf{J}}_{s}=\left\{\begin{array}{cccccc}
\frac{\partial \operatorname{Re}\left(\hat{F}_{t x, 0}\right)}{\partial \operatorname{Re}\left(\hat{X}_{A, 0}\right)} & \frac{\partial \operatorname{Re}\left(\hat{F}_{t x, 0}\right)}{\partial \operatorname{Im}\left(\hat{X}_{A, 0}\right)} & \frac{\partial \operatorname{Re}\left(\hat{F}_{t x, 0}\right)}{\partial \operatorname{Re}\left(\hat{Y}_{A, 0}\right)} & \ldots & \frac{\partial \operatorname{Re}\left(\hat{F}_{t x, 0}\right)}{\partial \operatorname{Re}\left(\hat{Z}_{A, n_{h}}\right)} & \frac{\partial \operatorname{Re}\left(\hat{F}_{t x, 0}\right)}{\partial \operatorname{Im}\left(\hat{Z}_{A, n_{h}}\right)} \\
\frac{\partial \operatorname{Im}\left(\hat{F}_{t x, 0}\right)}{\partial \operatorname{Re}\left(\hat{X}_{A, 0}\right)} & \frac{\partial \operatorname{Im}\left(\hat{F}_{t x, 0}\right)}{\partial \operatorname{Im}\left(\hat{X}_{A, 0}\right)} & \frac{\partial \operatorname{Im}\left(\hat{F}_{t x, 0}\right)}{\partial \operatorname{Re}\left(\hat{Y}_{A, 0}\right)} & \ldots & \frac{\partial \operatorname{Im}\left(\hat{F}_{t x, 1}\right)}{\partial \operatorname{Re}\left(\hat{Z}_{A, n_{h}}\right)} & \frac{\partial \operatorname{Im}\left(\hat{F}_{t x, 1}\right)}{\partial \operatorname{Im}\left(\hat{Z}_{A, n_{h}}\right)} \\
\vdots & \vdots & \vdots & \ddots & \vdots & \vdots \\
\frac{\partial \operatorname{Re}\left(\hat{F}_{n z, n_{h}}\right)}{\partial \operatorname{Re}\left(\hat{X}_{A, 0}\right)} & \frac{\partial \operatorname{Re}\left(\hat{F}_{n z, n_{h}}\right)}{\partial \operatorname{Im}\left(\hat{X}_{A, 0}\right)} & \frac{\partial \operatorname{Re}\left(\hat{F}_{n z, n_{h}}\right)}{\partial \operatorname{Re}\left(\hat{Y}_{A, 0}\right)} & \ldots & \frac{\partial \operatorname{Re}\left(\hat{F}_{n z, n_{h}}\right)}{\partial \operatorname{Re}\left(\hat{Z}_{A, n_{h}}\right)} & \frac{\partial \operatorname{Re}\left(\hat{F}_{n z, n_{h}}\right)}{\partial \operatorname{Im}\left(\hat{Z}_{A, n_{h}}\right)} \\
\frac{\partial \operatorname{Im}\left(\hat{F}_{n z, n_{h}}\right)}{\partial \operatorname{Re}\left(\hat{X}_{A, 0}\right)} & \frac{\partial \operatorname{Im}\left(\hat{F}_{n z, n_{h}}\right)}{\partial \operatorname{Im}\left(\hat{X}_{A, 0}\right)} & \frac{\partial \operatorname{Im}\left(\hat{F}_{n z, n_{h}}\right)}{\partial \operatorname{Re}\left(\hat{Y}_{A, 0}\right)} & \ldots & \frac{\partial \operatorname{Im}\left(\hat{F}_{n z, n_{h}}\right)}{\partial \operatorname{Re}\left(\hat{Z}_{A, n_{h}}\right)} & \frac{\partial \operatorname{Im}\left(\hat{F}_{n z, n_{h}}\right)}{\partial \operatorname{Im}\left(\hat{Z}_{A, n_{h}}\right)}
\end{array}\right\} .
$$

The Jacobian matrix is formulated in the frequency domain as required, whereas the friction forces are available in the time domain in the AFT procedure. The classical finite difference approach for the evaluation of the matrix is an extremely expensive and inefficient method, specially if a large number of temporal harmonics $(n)$ is chosen in order to capture the complex stick-slip phenomena, and in the case of multiple contact nodes such as to simulate the microslip behavior of the contact interface as presented in [43]. Therefore, a new method is needed to compute the Jacobian matrix efficiently for these cases. Cardona [33] outlined a general procedure for computing the Jacobian matrix from the Fourier transform of the time domain stiffness, damping and mass matrices of the system. Here this procedure has been extended to the nonlinear contact forces, and thus, the partial derivative of the $n^{\text {th }}$ Fourier coefficient of the nonlinear contact force with respect to the real and the imaginary part of the $j^{\text {th }}$ Fourier coefficient of the relative displacement is

$$
\begin{aligned}
\frac{\partial \hat{F}_{t x, n}}{\partial \operatorname{Re}\left(\hat{X}_{A, j}\right)} & =\frac{\partial \operatorname{Re}\left(\hat{F}_{t x, n}\right)}{\partial \operatorname{Re}\left(\hat{X}_{A, j}\right)}+\mathrm{i} \frac{\partial \operatorname{Im}\left(\hat{F}_{t x, n}\right)}{\partial \operatorname{Re}\left(\hat{X}_{A, j}\right)}=\frac{1}{N} \sum_{l=0}^{N-1} \frac{\partial F_{t x}(l)}{\partial \operatorname{Re}\left(\hat{X}_{A, j}\right)} e^{-\mathrm{i} n j 2 \pi / N} \\
& =\frac{1}{N} \sum_{l=0}^{N-1} \frac{\partial F_{t x}(l)}{\partial X_{A}(l)} \cos \left(j l \frac{2 \pi}{N}\right) e^{-\mathrm{i} n j 2 \pi / N}
\end{aligned}
$$

and

$$
\begin{aligned}
\frac{\partial \hat{F}_{t x, n}}{\partial \operatorname{Im}\left(\hat{X}_{A, j}\right)} & =\frac{\partial \operatorname{Re}\left(\hat{F}_{t x, n}\right)}{\partial \operatorname{Im}\left(\hat{X}_{A, j}\right)}+\mathrm{i} \frac{\partial \operatorname{Im}\left(\hat{F}_{t x, n}\right)}{\partial \operatorname{Im}\left(\hat{X}_{A, j}\right)}=\frac{1}{N} \sum_{l=0}^{N-1} \frac{\partial F_{t x}(l)}{\partial \operatorname{Im}\left(\hat{X}_{A, j}\right)} e^{-\mathrm{i} n j 2 \pi / N} \\
& =-\frac{1}{N} \sum_{l=0}^{N-1} \frac{\partial F_{t x}(l)}{\partial X_{A}(l)} \sin \left(j l \frac{2 \pi}{N}\right) e^{-\mathrm{i} n j 2 \pi / N}
\end{aligned}
$$

Note that the partial derivatives are required for all $n=0,1, \ldots, n_{h}$ with respect to all $j=0,1, \ldots, n_{h}$. Using the above expressions, the partial derivative of the Fourier coefficients of the contact forces are evaluated by differentiating the time history of contact forces with respect to the time domain relative displacement exclusively. Subsequently, by taking the FFT of the multiplication of the corresponding time derivative with sine or cosine functions. The interesting feature of this procedure is that it is quite well integrated with the AFT method, and thus the partial derivative of the Fourier coefficients of the contact forces is nicely evaluated in parallel to the contact forces with a small computation effort compared to the cost-intensive classical finite difference scheme. The partial derivative of the contact forces with respect to the relative displacements in the time domain can be determined using Eqs. (15) and (20)-(21). However, the time 
domain contact forces include the damper displacement term that is eliminated before differentiation as

$$
X_{D}(l-1)=X_{A}(l-1)-\frac{F_{t x}(l-1)}{K_{t}}
$$

and

$$
Y_{D}(l-1)=Y_{A}(l-1)-\frac{F_{t y}(l-1)}{K_{t}} .
$$

Note that the above equations are valid for all the contact states. Inserting Eqs. (33)-(34) into Eq. (20), the partial derivative of the contact force with respect to the Fourier coefficients of the relative displacement are

$$
\frac{\partial F_{t x}(l)}{\partial \operatorname{Re}\left(\hat{X}_{A, j}\right)}= \begin{cases}0 & \text { Separation } \\ K_{t}\left[\cos \left(j l \frac{2 \pi}{N}\right)-\cos \left(j(l-1) \frac{2 \pi}{N}\right)\right]+\frac{\partial F_{t x}(l-1)}{\partial \operatorname{Re}\left(\hat{X}_{A, j}\right)} & \text { Stick } \\ \mu F_{n z}(l)\left[\frac{1}{d(l)}\left(\cos \left(j l \frac{2 \pi}{N}\right)-\cos \left(j(l-1) \frac{2 \pi}{N}\right)+\frac{1}{K_{t}} \frac{\partial F_{t x}(l-1)}{\partial \operatorname{Re}\left(\hat{X}_{A, j}\right)}\right)\right. & \text { Slip } \\ -\frac{X_{r}(l)}{d(l)^{3}}\left(X_{r}(l)\left(\cos \left(j l \frac{2 \pi}{N}\right)-\cos \left(j(l-1) \frac{2 \pi}{N}\right)+\frac{1}{K_{t}} \frac{\partial F_{t x}(l-1)}{\partial \operatorname{Re}\left(\hat{X}_{A, j}\right)}\right)\right. & \\ \left.\left.+\frac{Y_{r}(l)}{K_{t}} \frac{\partial F_{t y}(l-1)}{\partial \operatorname{Re}\left(\hat{X}_{A, j}\right)}\right)\right] & \end{cases}
$$

$$
\frac{\partial F_{t x}(l)}{\partial \operatorname{Re}\left(\hat{Y}_{A, j}\right)}= \begin{cases}0 & \text { Separatic } \\ \frac{\partial F_{t x}(l-1)}{\partial \operatorname{Re}\left(\hat{Y}_{A, j}\right)} & \text { Stick } \\ \mu F_{n z}(l)\left[\frac { Y _ { r } ( l ) } { d ( l ) ^ { 3 } } \left(\frac{Y_{r}(l)}{K_{t}} \frac{\partial F_{t x}(l-1)}{\partial \operatorname{Re}\left(\hat{Y}_{A, j}\right)}\right.\right. & \text { Slip } \\ \left.\left.-X_{r}(l)\left(\cos \left(j l \frac{2 \pi}{N}\right)-\cos \left(j(l-1) \frac{2 \pi}{N}\right)+\frac{1}{K_{t}} \frac{\partial F_{t y}(l-1)}{\partial \operatorname{Re}\left(\hat{Y}_{A, j}\right)}\right)\right)\right] & \end{cases}
$$

Separation

and

$$
\frac{\partial F_{t x}(l)}{\partial \operatorname{Re}\left(\hat{Z}_{A, j}\right)}= \begin{cases}0 & \text { Separ } \\ \frac{\partial F_{t x}(l-1)}{\partial \operatorname{Re}\left(\hat{Z}_{A, j}\right)} & \text { Stick } \\ \mu F_{n z}(l)\left[\frac{Y_{r}(l)}{d(l)^{3}}\left[\frac{Y_{r}(l)}{K_{t}} \frac{\partial F_{t x}(l-1)}{\partial \operatorname{Re}\left(\hat{Z}_{A, j}\right)}-\frac{X_{r}(l)}{K_{t}} \frac{\partial F_{t y}(l-1)}{\partial \operatorname{Re}\left(\hat{Z}_{A, j}\right)}\right)\right] & \text { Slip, }\end{cases}
$$

where $X_{r}(l)=X_{A}(l)-X_{D}(l-1)$ and $Y_{r}(l)=Y_{A}(l)-Y_{D}(l-1)$. Note that the partial derivative of the time domain contact forces with respect to the imaginary part of relative displacement is evaluated by replacing the cosine with the sine function of opposite sign. Similar expressions are obtained for the contact forces in the tangential $y$-direction and the normal direction, see Appendix B.

\section{Solution method}

The resulting nonlinear algebraic equation with full-3D friction contact model often reveals the turning point bifurcation [44] caused by the variation of the normal load, especially in the case of the gap nonlinearities. In such cases, the standard Newton iteration step defined in Eq. (26) often fails to converge around the 
turning point, where the Jacobian matrix is close to singular. Therefore, a predictor-corrector continuation method [45] is applied in order to avoid the convergence problem, and consequently, the system of equations is augmented with an additional constraint equation, which may be a linear or spherical constraint. In this paper, the continuation method proposed by Keller (linear constraint) [45] is employed to solve the nonlinear EQM and augmented system of equations is

$$
\mathbf{e}(\tilde{\mathbf{q}}, \omega)=\mathbf{0} \text { and } \mathbf{v}_{\tilde{\mathbf{q}}}^{T}\left(\tilde{\mathbf{q}}-\tilde{\mathbf{q}}_{i+1}^{(1)}\right)+v_{\omega}\left(\omega-\omega_{i+1}^{(1)}\right)=0
$$

where $\mathbf{v}=\left\{\mathbf{v}_{\tilde{\mathbf{q}}}^{T}, v_{\omega}\right\}^{T}$ is the tangent vector and $\left(\tilde{\mathbf{q}}_{i+1}^{(1)}, \omega_{i+1}^{(1)}\right)$ is the initial guess of the solution, which is computed using the previously converged solution $\left(\tilde{\mathbf{q}}_{i}, \omega_{i}\right)$, the tangent vector and step-length $(\Delta s)$. Note that $\omega=n \Omega$ is a variable in the continuation method, since the solution is searched along the line orthogonal to the tangent vector and the curve, instead of at a fixed frequency step.

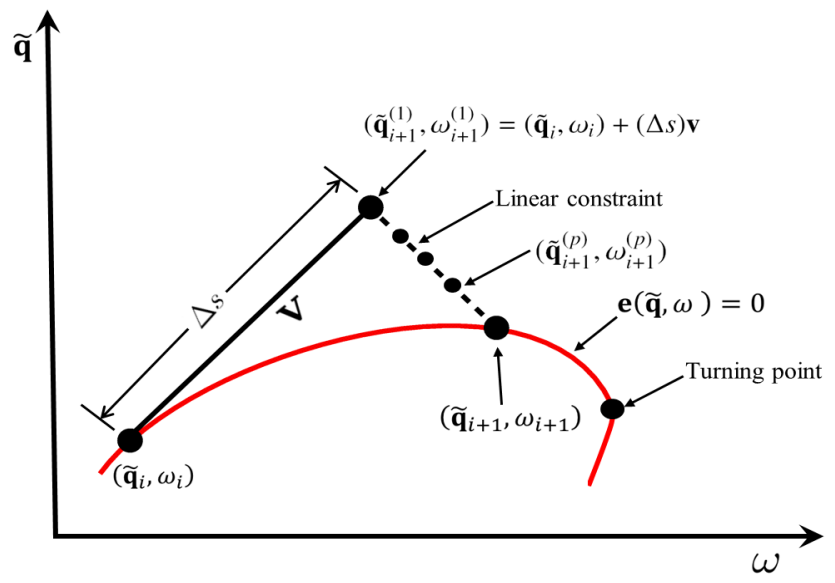

Figure 6: Continuation with linear constraint (Keller's method)

A large step-length around the turning point and on the steep branch of the curve may still lead to convergence problems and a too small step-length result in a costly computation. Linear scaling of variables [44] is known to reduce these problems since $\tilde{\mathbf{q}}$ and $\omega$ have different orders of magnitude. However, linear scaling has little effect and a good estimate of the step-length and adaptation method are also required in order to optimize the computation time and reduce the convergence problems. There are many ways to introduce step-length adaption, which is mainly based on the performance of the Newton iterations. For example, a simple scheme increases the step-length whenever few Newton iterations are needed to compute the last point, and conversely, the step-length is decreased when many iterations are required. Note that this simple adaptation scheme does not take particular care of the turning point and therefore the convergence problems around this point persist.

In this paper, in addition to the above adaptation scheme and scaling of variables, a method is proposed that facilitates a fine control of the step-length around the turning point based on the direction vector, $v_{\omega}$. It should be noted that the sign of $v_{\omega}$ changes around the turning point from positive to negative and vice versa, therefore as the continuation algorithm encounters $v_{\omega,(i-1)} * v_{\omega,(i)}<0$, it moves back by two or three steps and recalculates the solution with a step-length of $(1 / 10)^{\text {th }}$ of the currently in used. Moreover, an additional control strategy for the steep branch of the curve has also been implemented in the code, that is determined by the $l^{2}$-norm of $\mathbf{v}_{\tilde{\mathbf{q}}}$ and formulated as,

$$
\left\|v_{\tilde{\mathbf{q}}, i}\right\|>\alpha\left\|\mathbf{v}_{\tilde{\mathbf{q}}, i-1}\right\|
$$


where $\alpha$ has a constant value ranging $20-40$. If the above criteria are satisfied then the maximum steplength is restricted for those solution points. This helps in controlling the convergence failure around the resonance and on the steep portion of the curve. Implementing the above control strategy, a smaller steplength size is used around the turning point and on the steep branch of the curve to avoid convergence failure and branch switching, while on the other portion of the curve a large step-length is employed. Therefore, it optimizes the computation time while tracing the accurate dynamic behavior.

\section{Numerical results - applied to a test case shrouded blade}

The proposed method is applied on a simplified turbine blade model consisting of eight sectors, as shown in Fig. 7. Blades are coupled by an extended shroud and each sector is discretized into 814 elements with mid-side nodes using commercial FEM software ANSYS ${ }^{\circledR}$. One sector comprises 2719 nodes, 8157 DOFs and modal truncation is applied by keeping the lowest 50 mode shapes of the uncoupled sector in the computation of the FRF matrix. A point excitation $\hat{f}_{x, \mathrm{ext}}=\hat{f}_{\text {,ext }}=\hat{f}_{z, \mathrm{ext}}=1 \mathrm{~N}$ is used in all the following

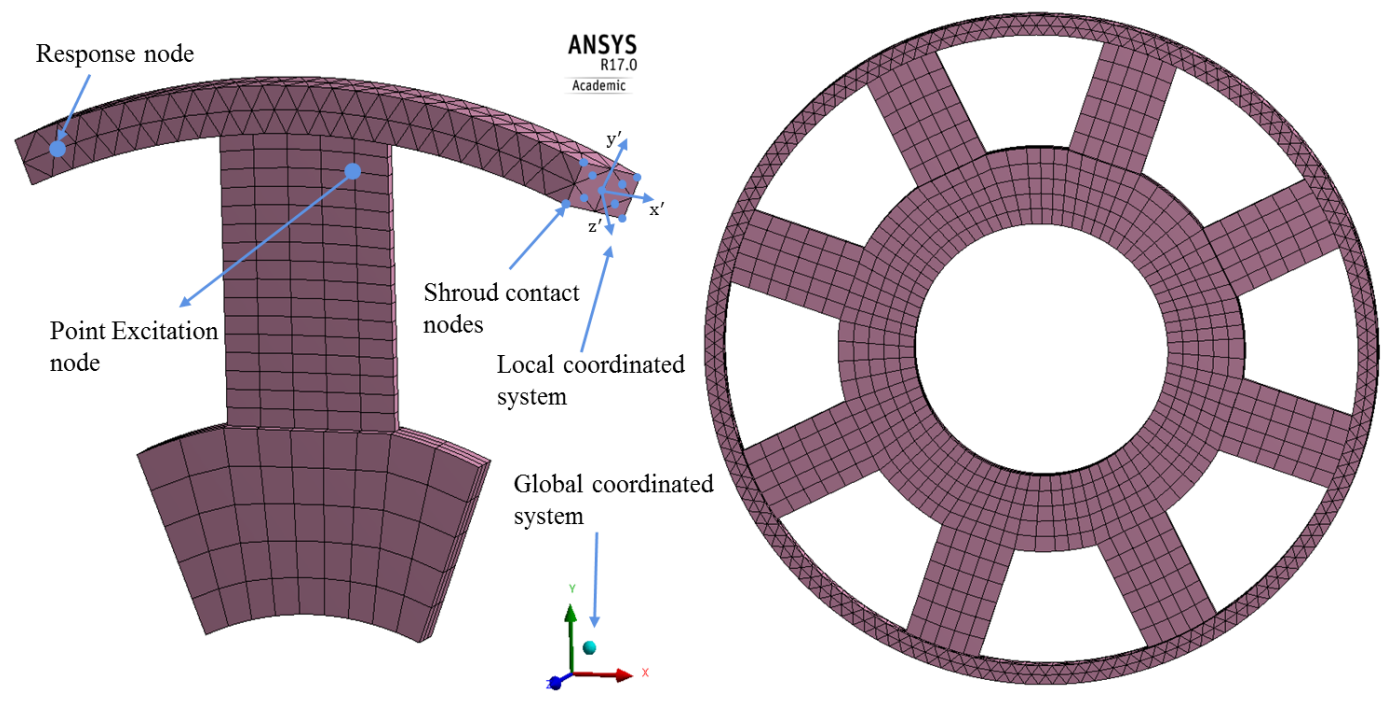

Figure 7: FEM model of a single sector and the full turbine bladed disk

examples to excite the structure. The excitation frequency is varied from $100 \mathrm{~Hz}$ to $220 \mathrm{~Hz}$ and internal structural damping is introduced as modal damping ratio and set to 0.001 for each mode. The nodal diameter (ND) map for the first six mode-families (MFs) and campbell diagram of the test case turbine blade are shown in Fig. 8. In the calculations performed, the steady state amplitude at the response node is computed as $\max \left(\sqrt{x(t)^{2}+y(t)^{2}+z(t)^{2}}\right)$ over one period, where $x(t), y(t)$ and $z(t)$ are the time domain displacements of the response node in the global coordinate system at the sought frequency. Since in general, the largest response amplitudes are found at the shroud, in order to study the effect of friction damping clearly, the response node is selected close to the shroud interface, see Fig. 7.

To demonstrate the necessity of the full-3D friction contact model and the computational efficiency of the derived Jacobian matrix, four examples are discussed in this section.

(i) In the first example, accuracy of the time-discrete scheme is compared with the time-continuous friction contact model and it has been shown that as the number of time steps $(N)$ increases, the relative error in the 
nonlinear response curve becomes negligible.

(ii) This example focuses on a key point of the paper, where the quasi-3D and the full-3D friction contact model are compared. It has been proved that a full-3D friction contact model is essential to analyze the complex contact motion of the shroud interface.

(iii) In this example, the effect of the shroud contact angle on the nonlinear forced response curve is presented. The obtained curve depicts that separation of the shroud contact interfaces that lead to the turning point bifurcation can be avoided by proper designing of the shroud angle.

(iv) The last example compares the computation time of the forced response curve while using the analytical Jacobian and the finite difference Jacobian in the full-3D friction contact model. A significant amount of time saving is obtained by using the analytical Jacobian with multiple contact nodes at the friction interface.

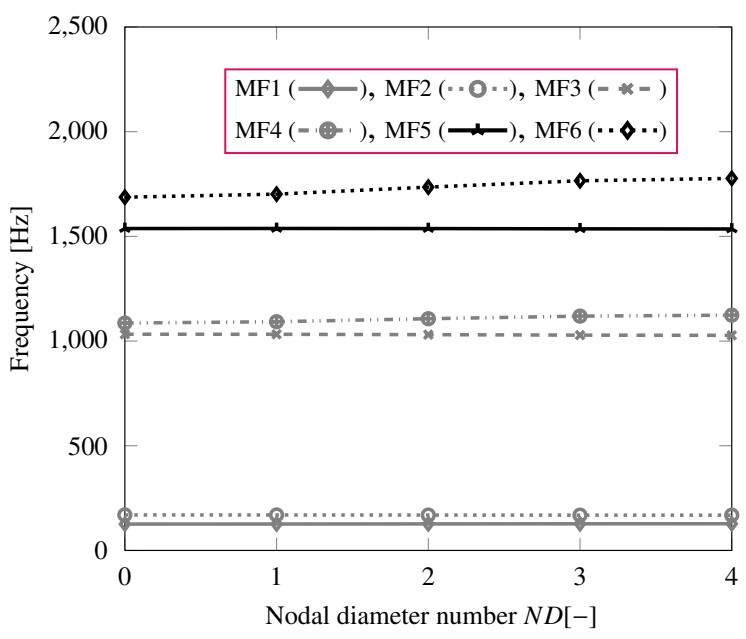

(a) Nodal diameter map

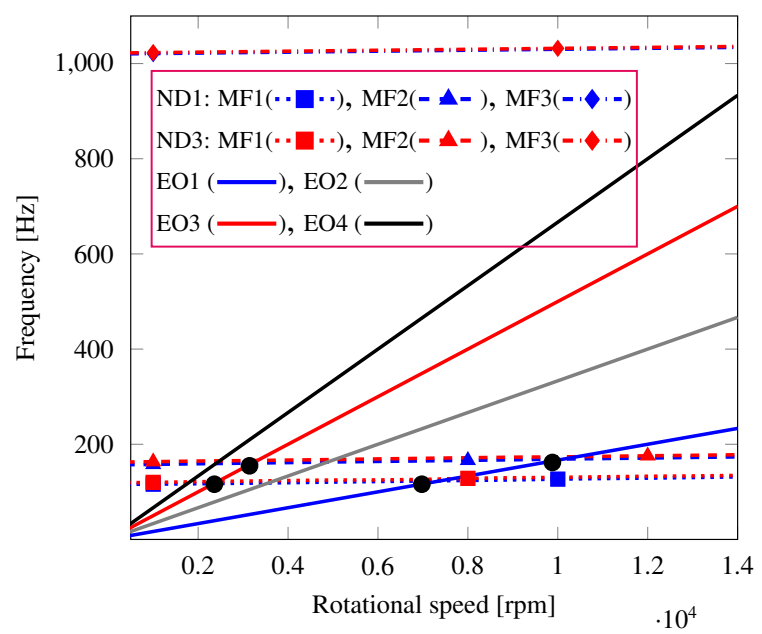

(b) Campbell diagram

Figure 8: Nodal diameter map and campbell diagram

Example 1 (Comparison of the time-continuous and the time-discrete scheme for a 1D-tangential motion and constant normal load friction contact model): As described in Section 1, the accuracy of the time-discrete (AFT) scheme is limited by the number of time steps $(N)$ used in the IFFT/FFT procedure. Therefore, response curves computed using the time-continuous and the AFT scheme are compared for 1D-tangential motion and constant load case. Engine excitation with the spatial periodicity of $m=3$, which corresponds to engine order3 (EO3) is used and only one harmonic is kept in the EQM. EO3 in the swept frequency range excites the first bending (MF1) and the first torsion (MF2) mode of ND3 (backward travelling wave), see Fig. 8b. The obtained nonlinear forced response and the error in the response curve for different values of $N$ are shown in Fig. 9. The relative error with respect to time-continuous solution is below $0.6 \%$ in all cases, which demonstrates that $N=64$ can be used to compute the response curve with satisfactory accuracy in this case. However, stick-slip motion for a full-3D contact model is rather complex compared to the 1D-tangential motion and constant normal load. Therefore, $N=512$ is used in the following examples. 


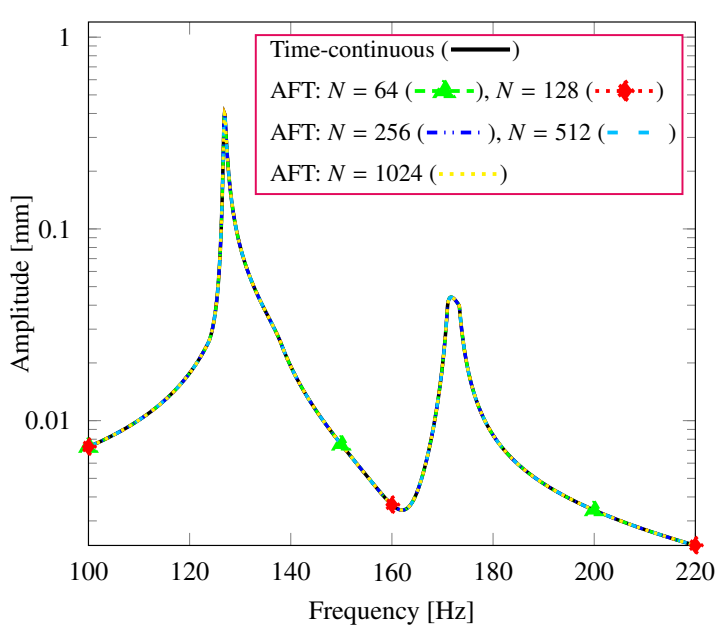

(a) Response curves for time-continuous and AFT scheme for different number of time steps $(N)$

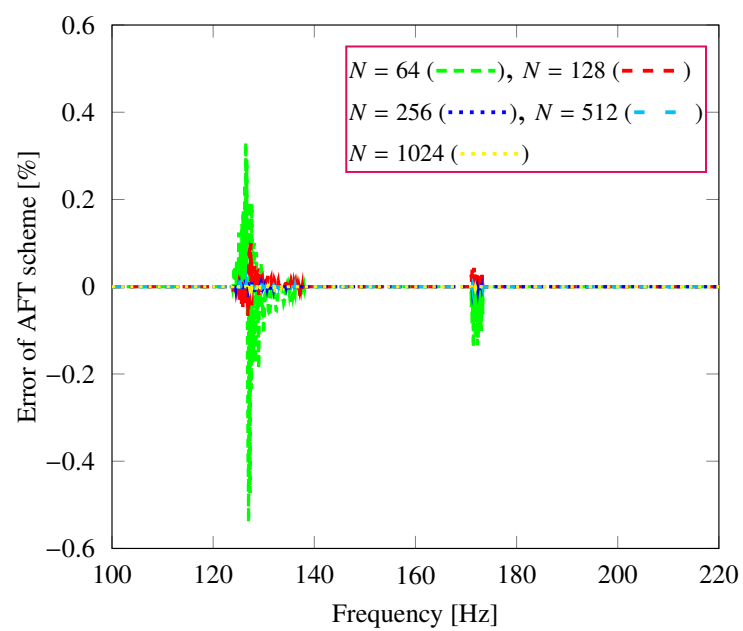

(b) Relative error in the amplitude of response curve

Figure 9: Comparison of time-continuous and time-discrete (AFT) scheme for 1D-tangential motion and constant normal $\operatorname{load}=1 \mathrm{~N}$

Example 2 (Comparison of the quasi-3D(uncoupled tangential motion) and full-3D(coupled tangential motion) friction contact model): To demonstrate the necessity of a full-3D friction contact model, response curves are compared by applying both contact models in two different local coordinate systems as depicted in Fig. 10.

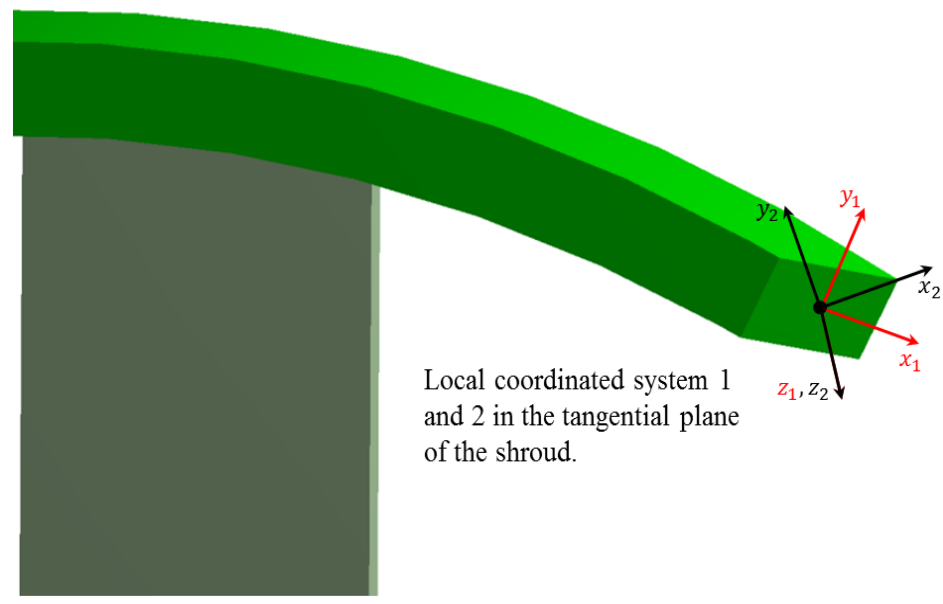

Figure 10: Local coordinate system 1 and 2 in the tangential plane of the shroud interface

In this example, a monofrequent excitation of EO1 is applied and five harmonics are considered in the MHBM formulation to capture the response accurately. The first harmonic will excite ND1 in backward travelling wave and the higher order harmonics (2..5) will excite ND2 in backward travelling wave, ND3 in both backward and forward travelling wave and ND4 in standing wave mode, see Fig. 8. However, in this 
case, the nonlinear forced response curve is dominated by MF1 and MF2 corresponding to ND1 only and contribution from higher harmonics is marginal in the swept frequency range, see Figs. 8b and 11.

The resulting nonlinear response curve and friction loop are plotted in Fig. 12 with varying normal preloads and gaps on the shroud interface, by applying the contact model (Section 3) in the local coordinate system 1 . The linear response of the system is obtained in the absence of friction contact. It can be seen that a little friction damping is obtained around the first bending mode (1F or MF1) for all the contact loads, whereas the first torsion mode (1T or MF2) is damped substantially. The 1F mode of ND1 corresponds to the inphase motion of the shroud interfaces, which means a small relative displacement is introduced at the interface in this mode and consequently little damping is observed. On the other hand, the 1T mode of ND1 corresponds to the out of phase motion of the shroud interfaces and therefore benefits significantly from friction damping. Moreover, there is an optimum preload around $0.25 \mathrm{~N}$ at which maximum friction damping is achieved as seen for the 1T mode. In addition, no visual difference in the response curves is observed between the uncoupled and coupled friction contact model in this case, which is a surprising result. A further investigation indicates that the relative motion in the shroud contact plane is a 1D tangential motion dominated along one axis, which explains why both contact models give similar results. Furthermore, in Fig. $12 \mathrm{~b}$ the friction loops in $x_{1}$ and $y_{1}$-directions are plotted which clearly shows that motion is dominated along the $x_{1}$-direction exclusively. Note that friction loop is plotted in the local coordinate system 1 of the shroud interface and the friction loop is captured while computing the nonlinear friction force in the time domain.

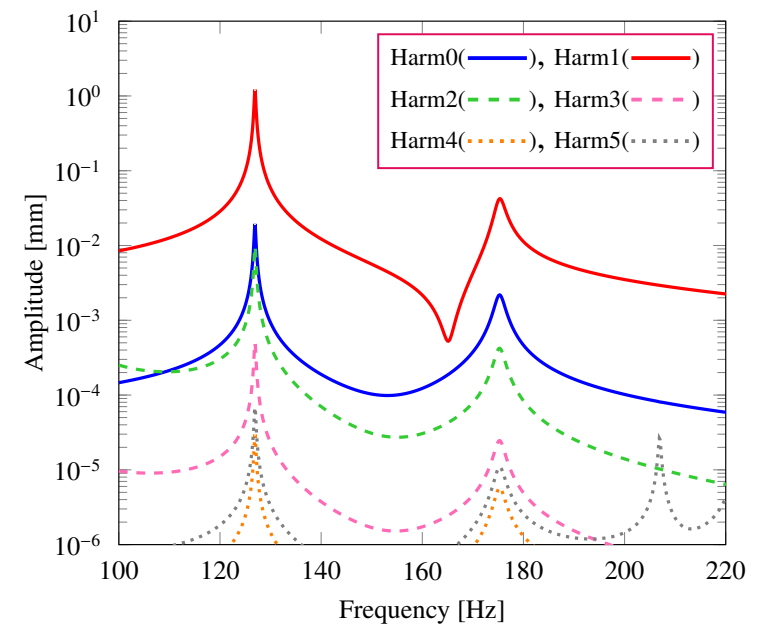

(a) Harmonic components at $N_{0}=0 \mathrm{~N}$

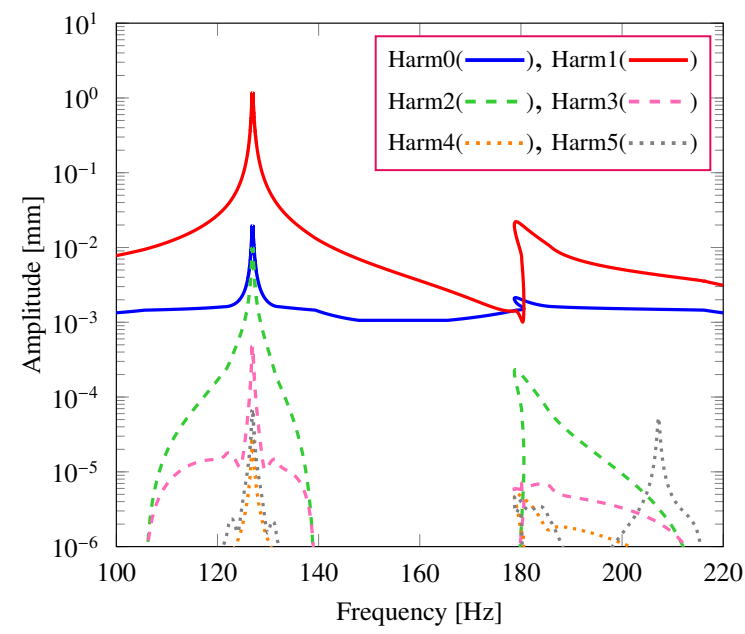

(b) Harmonic components at $N_{0}=0.15 \mathrm{~N}$

Figure 11: Harmonic components of the nonlinear response curve using full-3D contact model

In Fig. 13, the contact model is applied in the local coordinate system 2 (Fig. 10), which is at an angle of $45^{\circ}$ from the local coordinate system 1 . In the new local coordinate system, relative motion of the shroud interface is still a 1D tangential motion, however the motion is not dominated along one axis. Therefore, a significant difference in the response curves for the uncoupled and coupled contact models is seen. The transition from pure sticking to stick-slip takes place at a smaller magnitude of the relative displacement in the case of the coupled formulation compared to the uncoupled formulation. This happens because the stick-slip transition depends on the magnitude of the relative displacement in the tangential plane for the case of the coupled formulation while in the case of the uncoupled formulation the displacement components on each tangential axis are independently considered which delays the transition. As a result, the uncoupled 
formulation underestimates or overestimates friction damping as shown in Fig. 13.

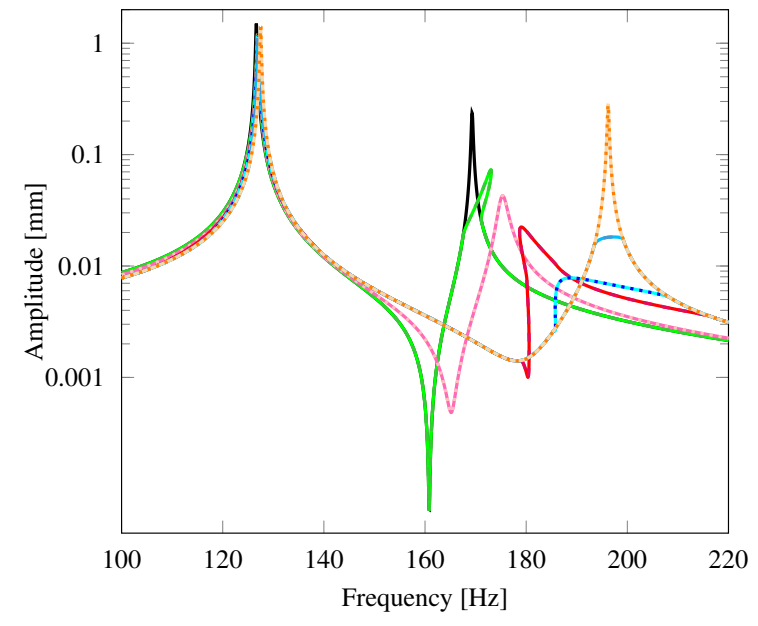

(a) Linear response( $\longrightarrow$ ), nonlinear response with gap $=$ $0.03 \mathrm{~mm}(-,---), \quad N_{0}=0 \mathrm{~N}(\square, \cdots \cdots), N_{0}=$ $0.15 \mathrm{~N}(\square,---), \quad N_{0}=0.25 \mathrm{~N}(-, \cdots \cdots), \quad N_{0}=$ $1 \mathrm{~N}(-,---)$ and $N_{0}=20 \mathrm{~N}(-, \cdots \cdots)$

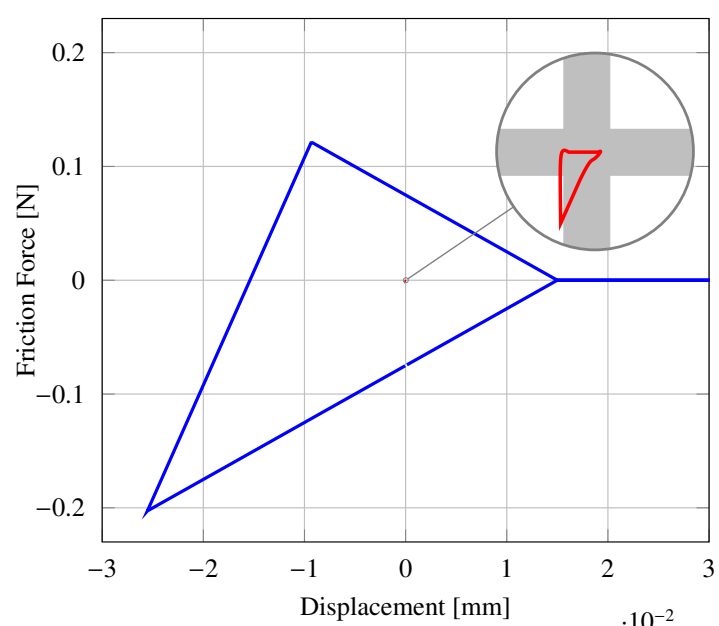

(b) Friction loop in $x_{1}$-direction( - ) and $y_{1}$-direction(---) in the shroud local coordinate system 1

Figure 12: (a) Response curve for the full-3D (solid line) and quasi-3D (dashed line) contact model and (b) Friction loop for the full-3D contact model at $178.7 \mathrm{~Hz}$ and $0.15 \mathrm{~N}$ normal load in the local coordinate system 1

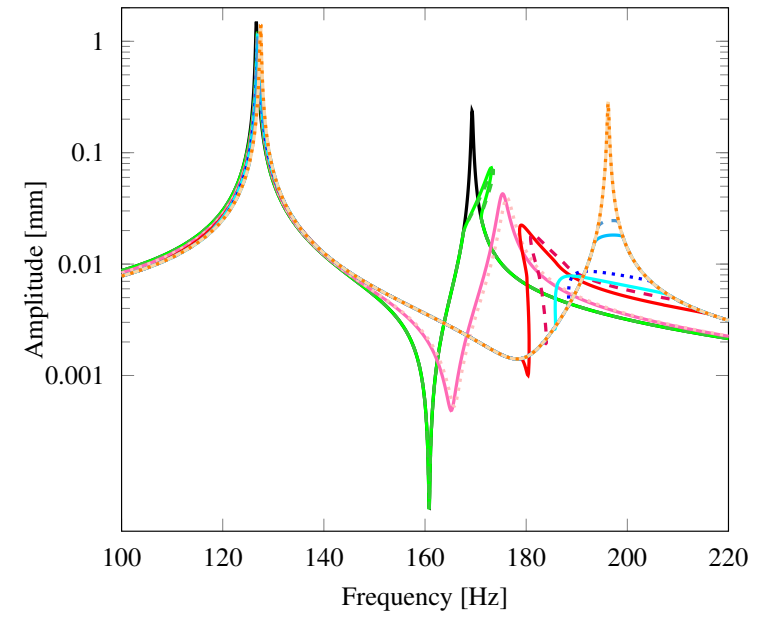

(a) Linear response (-), nonlinear response with gap = $0.03 \mathrm{~mm}(-,---), \quad N_{0}=0 \mathrm{~N}(\square, \cdots \cdots), \quad N_{0}=$ $0.15 \mathrm{~N}(-,---), \quad N_{0}=0.25 \mathrm{~N}(-, \cdots \cdots), \quad N_{0}=$ $1 \mathrm{~N}(-,---)$ and $N_{0}=20 \mathrm{~N}(-, \cdots \cdots)$

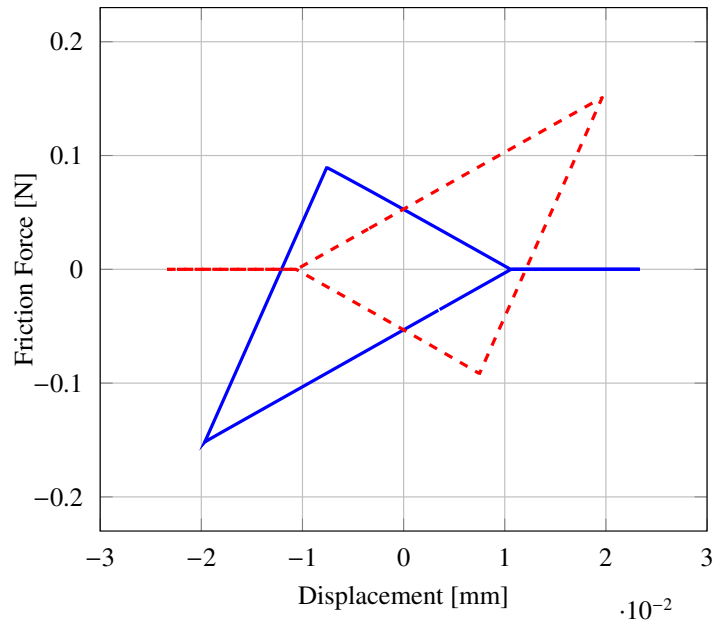

(b) Friction loop in $x_{2}$-direction( $(-)$ and $y_{2}$-direction(- - - ) in the shroud local coordinate system 2

Figure 13: (a) Response curve for the full-3D (solid line) and quasi-3D (dashed line) contact model and (b) Friction loop for the full-3D contact model at $178.7 \mathrm{~Hz}$ and $0.15 \mathrm{~N}$ normal load in the local coordinate system 2 
The above discussion reveals that if the tangential motion in the local shroud coordinate system is not dominated along one axis and in the case of 2D-planar motion, the coupled contact model should be considered for the computation of the optimum normal preload. Furthermore, in case the motion occurs along one axis in the shroud contact plane then the uncoupled formulation can be used since the uncoupled formulation gives a computational advantage as discussed in example 4 . However, it should be noted that the direction of the relative motion in the shroud contact plane is seldom known in advance.

Example 3 (Effect of the shroud geometry on the nonlinear forced response curve): In Fig. 10, the shroud tangential plane is at an angle of $45^{\circ}$ from the radial plane of the turbine bladed disk. The angle with the radial plane is changed from $0-90$ degree in order to investigate the influence of the shroud contact geometry. The contact model is applied in the local coordinate system 1, while all other parameters are as in example 2. The obtained result for the $1 \mathrm{~T}$ mode with two different normal load values is shown in Fig. 14. The curves clearly demonstrate that a small shroud angle leads to turning point bifurcation due to separation of the contact interface while a significant amount of damping is observed for higher shroud angles $\left(>45^{\circ}\right)$. The nonlinear peak amplitude is slightly higher than the linear response for the shroud angle 0 degree. Therefore, to achieve a good amount of friction damping the shroud angle should be greater than $45^{\circ}$ in this case. Note that the behavior of the nonlinear response curve is similar for both values of normal load.

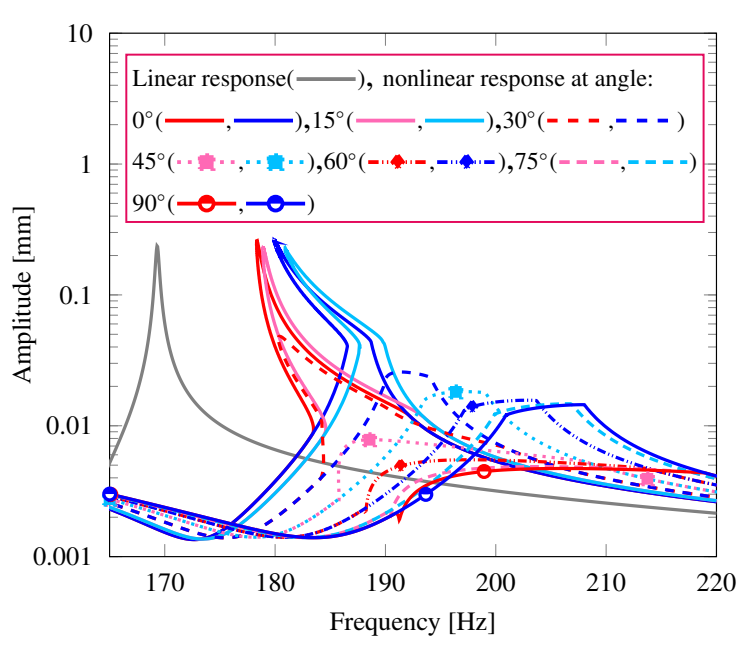

(a) Effect of shroud angle on the forced response curve

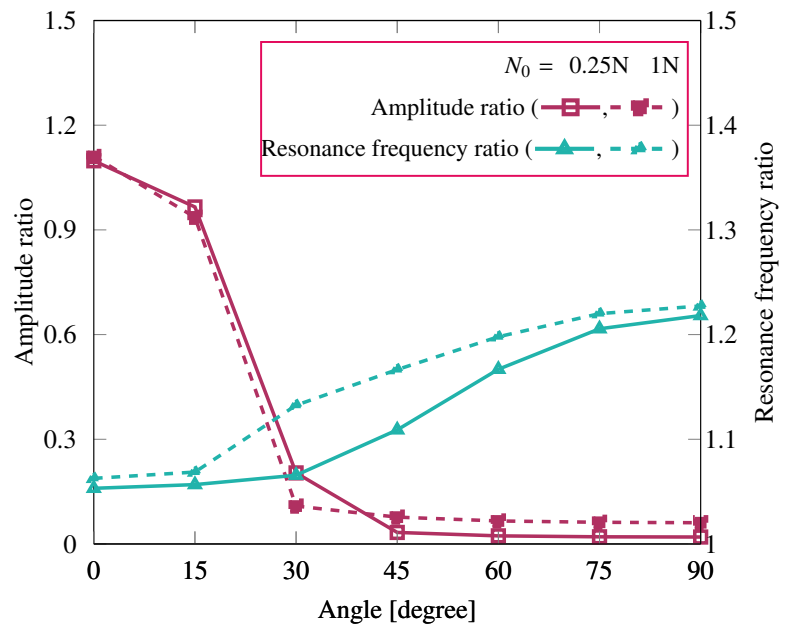

(b) Effect of shroud angle on the peak amplitude and resonance frequency

Figure 14: (a) Forced response curve with variation of shroud geometry at two different normal loads $(0.25 \mathrm{~N}, 1 \mathrm{~N})(\mathrm{b}) \mathrm{The}$ ratio of the nonlinear response to the linear response for different values of the shroud angle

Example 4 (Comparison of the computational time which is defined as the entire computing time for a complete FRF): Normalized computation time plots are shown in Figs. 15 and 16, where the maximum computational time is set to a value of 10 and all other values are normalized accordingly. In Fig. 15, the computation time for the coupled and uncoupled formulation are compared, and as indicated before the uncoupled formulation allows a time saving of $40 \%$ compared to the coupled formulation for a single contact node. Note that the computation time for the coupled formulation is higher due to the number of function evaluations not due to the convergence problem, since in the presented result the number of iterations performed in both cases is equal. 


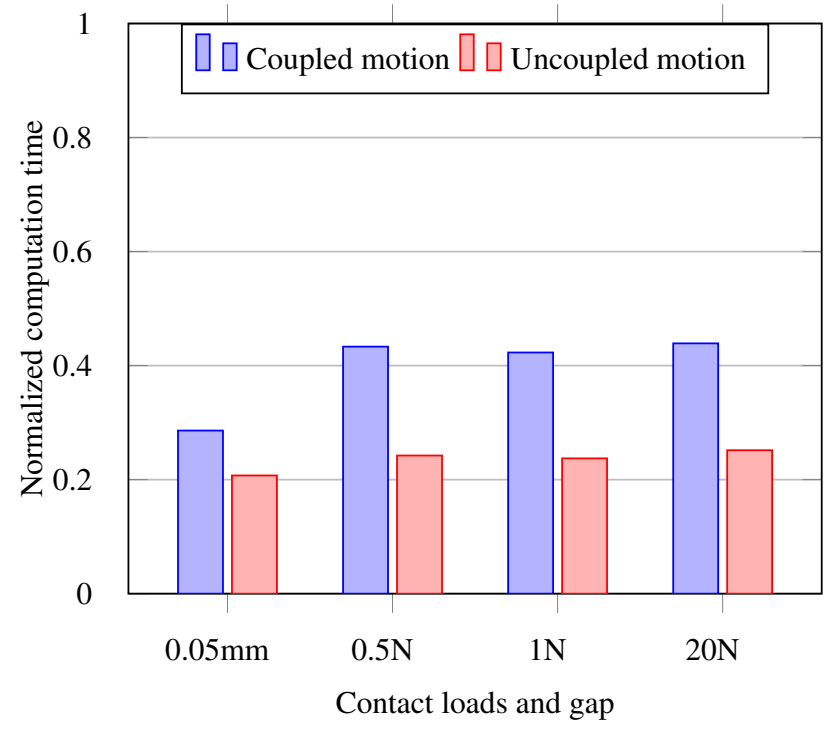

Figure 15: Comparison of the computation time for the coupled and uncoupled contact model using analytical Jacobian and a single contact node

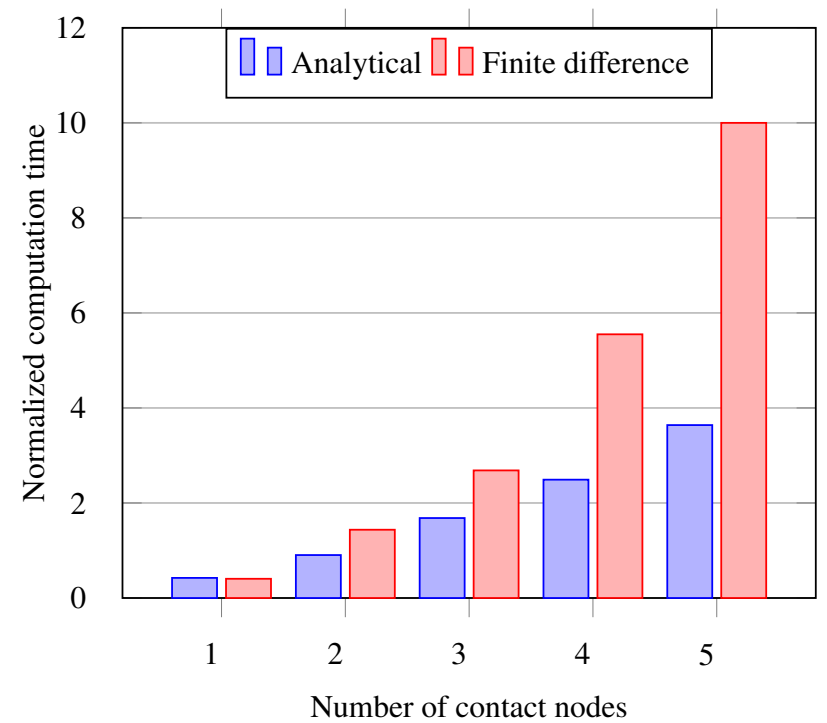

Figure 16: Comparison of the computation time for the analytical and finite difference Jacobian for different numbers of contact nodes using coupled contact model

Further, in Fig. 16, a comparison of the computation time between the analytical Jacobian and the classical finite difference method is shown. The plot clearly demonstrates that as the number of contact nodes increases the computational time using the finite difference Jacobian increases exponentially whereas the computational time for the analytical Jacobian has a linear relationship with the number of contact nodes. For a single contact node, the computational time for the analytical Jacobian and finite difference method are 
almost same. However as the number of contact nodes increases, the analytical method leads to a substantial computational time saving. Moreover, this comparison also makes it evident that the most time consuming step in solving a large set of nonlinear algebraic equations is the evaluation of the Jacobian matrix since a change in the Jacobian computation method leads to a large reduction in computational time.

Finally, to compare the error between the analytical and finite difference Jacobian matrices, the error graphs are plotted in Fig. 17 for different values of normal load and with varying size of FFT $(N)$. The error here is defined as $\max \left(\right.$ norm $\left.\left(\mathrm{J}_{\text {analytical }}-\mathrm{J}_{\text {finite-difference }}\right) / \operatorname{norm}\left(\mathrm{J}_{\text {analytical }}\right)\right)$ of the analyzed frequency range. The error decreases as $N$ increases and for $N \geq 512$, the error is below $0.7 \%$. Moreover, in some cases (eg. $N_{0}=0.5 \mathrm{~N}$ ) the error is quite low for lower values of $\mathrm{N}$. This is perhaps due the cancellation of error in the finite difference approach and in the analytical method. Based on the above results and discussion it is evident that the analytical method is quite fast compare to the finite difference approach and $N=512$ is sufficient for these calculations.

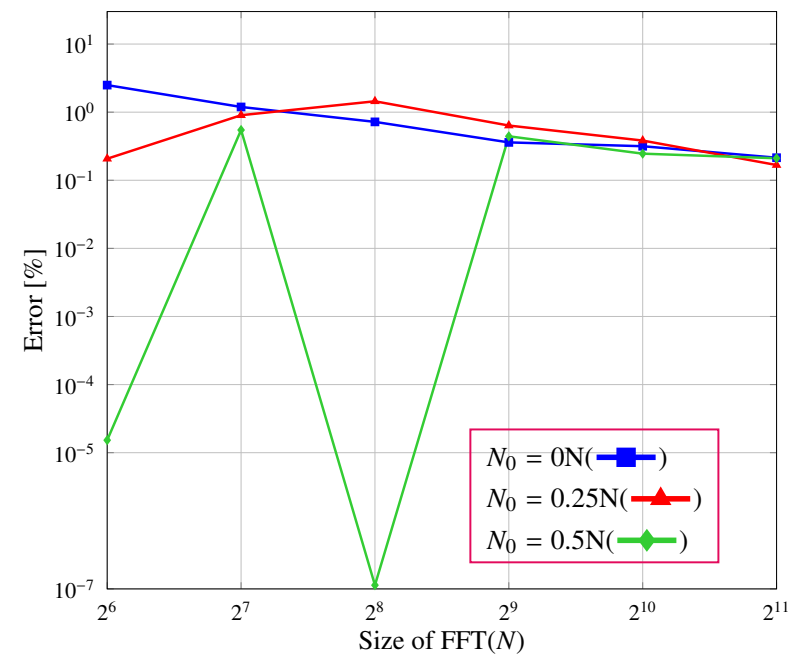

Figure 17: Error in the Jacobian matrix for different values of normal load $\left(N_{0}\right)$ and size of FFT $(N)$

\section{Conclusions}

In this paper, a 3D time-discrete friction contact model is reformulated and an analytical expression to compute its Jacobian matrix is derived. With this expression the Jacobian matrix is calculated in parallel with the computation of the nonlinear contact force in the AFT framework leading to a more efficient approach than to the classical finite difference approach. The proposed method is successfully implemented on a turbine bladed disk and a comparison between quasi-3D and full-3D contact model is presented. The numerical investigations show that the quasi-3D and full-3D friction contact models result in similar results if tangential motion in the shroud contact plane occurs mainly along one axis, whereas if the tangential motion is either $2 \mathrm{D}$ or $1 \mathrm{D}$ but not dominated along one axis in the chosen shroud local coordinate system, then the quasi-3D formulation leads to an over and underestimation of friction damping. Since the motion kinematics in the shroud contact plane is seldom known in advance, therefore the full-3D friction contact model is essential to accurately predict the nonlinear forced response of a real turbine blade disk.

The computation time of the analytical Jacobian is compared with the finite difference method. The comparison shows that the computational time with a single contact node is equal for both methods; however, 
as the number of contact nodes increases a substantial amount of time saving (70\% with 5 contact nodes) is achieved by using the analytical method. Therefore, it can be argued that the proposed method is more beneficial for the case of mistuned assemblies and for the simulation of microslip behavior using multiple contact nodes at interface. In addition, it is also noticed that the quasi-3D formulation requires $40 \%$ less time compared to the full-3D formulation, therefore the quasi-3D formulation should be used where applicable.

\section{Acknowledgements}

This research is funded by the Swedish Energy Agency, Siemens Industrial Turbomachinery AB, GKN Aerospace and the Royal Institute of Technology through the Swedish research program TurboPower, the support of which is gratefully acknowledged.

\section{Appendix Equation details}

Appendix A. Application of the Kronecker product in converting complex arithmetic to real arithmetic

- Kronecker product of a $(m \times n)$ matrix $\mathbf{A}$ with a matrix $\mathbf{B}$ is defined as

$$
\mathbf{A} \otimes \mathbf{B}=\left[\begin{array}{ccc}
a_{11} \mathbf{B} & \cdots & a_{1 n} \mathbf{B} \\
\vdots & \ddots & \vdots \\
a_{m 1} \mathbf{B} & \cdots & a_{m n} \mathbf{B}
\end{array}\right]
$$

- Real arithmetic of a complex vector $\left([c, d]^{\mathrm{T}}\right)$ can be obtained using a Kronecker product as

$$
\text { Real arithmetic of }\left[\begin{array}{l}
c \\
d
\end{array}\right]=\left[\begin{array}{l}
\operatorname{Re}(c) \\
\operatorname{Im}(c) \\
\operatorname{Re}(d) \\
\operatorname{Im}(d)
\end{array}\right]=\operatorname{Re}\left\{\left[\begin{array}{l}
c \\
d
\end{array}\right] \otimes\left[\begin{array}{c}
1 \\
-\mathrm{i}
\end{array}\right]\right\}
$$

- Real arithmetic of a $(2 \times 2)$ matrix can be obtained using a Kronecker product as

$$
\text { Real arithmetic of }\left[\begin{array}{ll}
a & c \\
b & d
\end{array}\right]=\left[\begin{array}{cccc}
\operatorname{Re}(a) & -\operatorname{Im}(a) & \operatorname{Re}(c) & -\operatorname{Im}(c) \\
\operatorname{Im}(a) & \operatorname{Re}(a) & \operatorname{Im}(c) & \operatorname{Re}(c) \\
\operatorname{Re}(b) & -\operatorname{Im}(b) & \operatorname{Re}(d) & -\operatorname{Im}(d) \\
\operatorname{Im}(b) & \operatorname{Re}(b) & \operatorname{Im}(d) & \operatorname{Re}(d)
\end{array}\right]=\operatorname{Re}\left\{\left[\begin{array}{ll}
a & c \\
b & d
\end{array}\right] \otimes\left[\begin{array}{cc}
1 & \mathrm{i} \\
-\mathrm{i} & 1
\end{array}\right]\right\}
$$

Above expressions are valid for $(m \times 1)$ vector and $(m \times m)$ matrix as well.

Appendix B. Partial derivative of the time domain contact forces with respect to the Fourier coefficient of the relative displacement

- The partial derivative of the tangential contact force in $y$-direction 


$$
\begin{aligned}
& \frac{\partial F_{t y}(l)}{\partial \operatorname{Re}\left(\hat{X}_{A, j}\right)}= \begin{cases}0 & \text { Separati } \\
\frac{\partial F_{t y}(l-1)}{\partial \operatorname{Re}\left(\hat{X}_{A, j}\right)} & \text { Stick } \\
\mu F_{n z}(l)\left[\frac { X _ { r } ( l ) } { d ( l ) ^ { 3 } } \left(\frac{X_{r}(l)}{K_{t}}\left(\frac{\partial F_{t y}(l-1)}{\partial \operatorname{Re}\left(\hat{X}_{A, j}\right)}\right)\right.\right. & \text { Slip } \\
\left.\left.-Y_{r}(l)\left(\cos \left(j l \frac{2 \pi}{N}\right)-\cos \left(j(l-1) \frac{2 \pi}{N}\right)+\frac{1}{K_{t}} \frac{\partial F_{t x}(l-1)}{\partial \operatorname{Re}\left(\hat{X}_{A, j}\right)}\right)\right)\right] & \end{cases} \\
& \frac{\partial F_{t y}(l)}{\partial \operatorname{Re}\left(\hat{Y}_{A, j}\right)}= \begin{cases}0 & \text { Separation } \\
K_{t}\left(\cos \left(j l \frac{2 \pi}{N}\right)-\cos \left(j(l-1) \frac{2 \pi}{N}\right)\right)+\frac{\partial F_{t y}(l-1)}{\partial \operatorname{Re}\left(\hat{Y}_{A, j}\right)} & \text { Stick } \\
\mu F_{n z}(l)\left[\frac{1}{d(l)}\left(\cos \left(j l \frac{2 \pi}{N}\right)-\cos \left(j(l-1) \frac{2 \pi}{N}\right)+\frac{1}{K_{t}} \frac{\partial F_{t y}(l-1)}{\partial \operatorname{Re}\left(\hat{Y}_{A, j}\right)}\right)\right. & \text { Slip } \\
-\frac{Y_{r}(l)}{d(l)^{3}}\left(Y_{r}(l)\left(\cos \left(j l \frac{2 \pi}{N}\right)-\cos \left(j(l-1) \frac{2 \pi}{N}\right)+\frac{1}{K_{t}} \frac{\partial F_{t y}(l-1)}{\partial \operatorname{Re}\left(\hat{Y}_{A, j}\right)}\right)\right. & \\
\left.\left.+\frac{X_{r}(l)}{K_{t}} \frac{\partial F_{t x}(l-1)}{\partial \operatorname{Re}\left(\hat{Y}_{A, j}\right)}\right)\right] & \end{cases} \\
& \frac{\partial F_{t y}(l)}{\partial \operatorname{Re}\left(\hat{Z}_{A, j}\right)}= \begin{cases}0 & \text { Separation } \\
\frac{\partial F_{t y}(l-1)}{\partial \operatorname{Re}\left(\hat{Z}_{A, j}\right)} & \text { Stick } \\
\mu F_{n z}(l)\left[\frac{X_{r}(l)}{d(l)^{3}}\left(\frac{X_{r}(l)}{K_{t}} \frac{\partial F_{t y}(l-1)}{\partial \operatorname{Re}\left(\hat{Z}_{A, j}\right)}-\frac{Y_{r}(l)}{K_{t}} \frac{\partial F_{t x}(l-1)}{\partial \operatorname{Re}\left(\hat{Z}_{A, j}\right)}\right)\right] & \text { Slip }\end{cases}
\end{aligned}
$$

- The partial derivative of the normal contact forces

$$
\begin{gathered}
\frac{\partial F_{n z}(l)}{\partial \operatorname{Re}\left(\hat{X}_{A, j}\right)}=\frac{\partial F_{n z}(l)}{\partial \operatorname{Re}\left(\hat{Y}_{A, j}\right)}=0 \text { For all states } \\
\frac{\partial F_{n z}(l)}{\partial \operatorname{Re}\left(\hat{Z}_{A, j}\right)}= \begin{cases}0 & \text { Separation } \\
K_{n} \cos \left(j l \frac{2 \pi}{N}\right) & \text { Contact }\end{cases}
\end{gathered}
$$

\section{References}

[1] J. H. Griffin, A review of friction damping of turbine blade vibration, International Journal of Turbo and Jet Engines 7 (1990) 297-307.

[2] A. V. Srinivasan, D. G. Cutts, Dry friction damping mechanisms in engine blades, ASME Journal of Engineering for Power 105 (1983) 332-341.

[3] A. Przekop, S. A. Rizzi, A reduced order method for predicting high cycle fatigue of nonlinear structures, Computers and Structures 84 (2006) 1606-1618.

[4] W. Ostachowicz, Forced vibrations of a beam including dry friction dampers, Computers \& Structures 33 (1989) 851-858. 
[5] C. Siewert, L. Panning, J. Wallaschek, C. Richter, Multiharmonic forced response analysis of a turbine blading coupled by nonlinear contact forces, ASME Journal of Engineering for Gas Turbines and Power 132 (2010) 082501-1 - 082501-9.

[6] B. D. Yang, J. J. Chen, C. H. Menq, Prediction of resonant response of shrouded blades with threedimensional shroud constraint, ASME Journal of Engineering for Gas Turbines and Power 121 (1999) $523-529$.

[7] B. D. Yang, C. H. Menq, Characterization of 3d contact kinematics and prediction of resonant response of structures having 3d frictional constraint., Journal of Sound and Vibration 217(5) (1998) 909-925.

[8] O. Poudou, C. Pierre, Hybrid frequency-time domain methods for the analysis of complex structural systems with dry friction damping, in: 44th AIAA/ASME/ASCE/AHS Structural Dynamics and Materials Conference, 7-10 April 2003, Norfolk, Virginia, 2003.

[9] J. H. Griffin, Friction damping of resonant stresess in gas turbine engine airfoils, Journal of Engineering for Power 102 (1980) 329-333.

[10] A. Sinha, J. H. Griffin, Effects of static friction on the forced response of frictionally damped turbine blades, ASME Journal of Engineering for Gas Turbine and Power 106 (1984) 65-69.

[11] C. H. Menq, J. H. Griffin, J. Bielak, The forced responses of shrouded fan stages, ASME Journal of Vibration, Acoustic, Stress and Reliability in Design 108 (1986) 50-55.

[12] T. M. Cameron, J. H. Griffin, R. E. Kielb, T. M. Hoosac, An integrated approach for friction damper design, ASME Journal of Vibration and Acoustic 112 (1990) 175-182.

[13] C. H. Menq, J. H. Griffin, J. Bielak, The influence of a variable normal load on the forced vibration of a frictionally damped structure, ASME Journal of Engineering for Gas Turbines and Power 108 (1986) 300-305.

[14] C. H. Menq, P. Chidamparam, J. H. Griffin, Friction damping of two-dimensional motion and its application in vibrational control, Journal of Sound and Vibration 144(3) (1991) 427-447.

[15] B. D. Yang, C. H. Menq, Modelling of friction contact and its application to the design of shroud contact, ASME Journal of Engineering for Gas Turbines and Power 119 (1997) 958-963.

[16] B. D. Yang, C. H. Menq, Nonlinear spring resistance and friction damping of frictional constraints having two-dimensional motion, Journal of Sound and Vibration 217 (1998) 127-143.

[17] B. D. Yanq, C. H. Menq, Characterization of contact kinematics and application to the design of wedge damper in turbomachinery blading: Part1 - stick-slip contact kinematics, ASME Journal of Engineering for Gas Turbine and Power 120 (1997) 410-417.

[18] B. D. Yanq, C. H. Menq, Characterization of contact kinematics and application to the design of wedge dampers in turbomachinery application: Part2 - prediction of forced response and experimental verification, ASME Journal of Engineering for Gas Turbine and Power 120 (1998) 418-423.

[19] E. P. Petrov, D. J. Ewins, Analytical formulation of friction interface elements for analysis of nonlinear multiharmonic vibrations of bladed disks, ASME Journal of Turbomachinery 125 (2003) 364-371. 
[20] M. Krack, L. Panning, J. Wallaschek, A high-order harmonic balance method for systems with distinct states, Journal of Sound and Vibration 332 (2013) 5476 - 5488.

[21] C. Siewert, M. Krack, L. Panning, J. Wallaschek, The nonlinear analysis of the multiharmonic forced response of coupled turbine blading, in: ISROMAC12-2008-20219, 2008.

[22] K. Y. Sanliturk, D. J. Ewins, Modelling two-dimensional friction contact and its application using harmonic balance method, Journal of Sound and Vibration 193(2) (1996) 511-523.

[23] S. Yajie, H. Jie, Z. Zigen, Forced response analysis of shrouded blades by an alternating frequency/time domain method, in: Paper GT2006-90595, Proceedings of ASME Turbo Expo 2006, May 8-11, Barcelona, Spain, 2006.

[24] W. Gu, Z. Xu, 3d numerical friction contact model and its application to nonlinear blade damping, in: Paper GT2010-22292, Proceedings of ASME Turbo Expo 2010, June 14-18, Glasgow, UK, 2010.

[25] W. Gu, Z. Xu, Y. Liu, A method to predict the nonlinear vibratory response of bladed disc system with shrouded dampers, in: Proceeding of the IMechE Vol. 226 Part C: Journal of Mechanical Engineering Science, 2011.

[26] T. M. Cameron, J. H. Griffin, An alternating frequency/time domain method for calculating the steady state response of nonlinear dynamics systems, Journal of Applied Mechanics 56 (1989) 149-154.

[27] A. LaBryer, P. Attar, A harmonic balance approach for large-scale problems in nonlinear structural dynamics, Computers and Structures 88 (2010) 1002-1014.

[28] R. Hayes, S. P. Marques, Prediction of limit cycle oscillations under uncertainty using a harmonic balance method, Computers and Structures 148 (2015) 1-13.

[29] E. P. Petrov, D. J. Ewins, Generic friction models for time-domain vibration analysis of bladed disks, ASME Journal of Engineering for Gas Turbine and Power 126 (2004) 184-192.

[30] P. Deuflhard, Newton methods for nonlinear problems, Vol. 35, Springer, 2000.

[31] J. Guillen, Studies of the dynamics of dry - friction - damped blade assemblies, Ph.D. thesis, University of Michigan-Ann Arbor (1999).

[32] J. M. Borrajo, S. Zucca, M. M. Gola, Analytical formulation of the jacobian matrix for nonlinear calculation of the forced response of turbine blade assemblies with wedge friction dampers, International Journal of Non-Linear Mechanics 41 (2006) 1118-1127.

[33] A. Cardona, T. Coune, A. Lerusse, M. Geradin, A multiharmonic method for nonlinear vibration analysis, International Journal for Numerical Methods in Engineering 37 (1994) 1593-1608.

[34] D. L. Thomas, Dynamics of rotationally periodic structures, International Journal for Numerical Methods in Engineering 14 (1979) 81-102.

[35] E. P. Petrov, A method for use of cyclic symmetry properties in analysis of nonlinear multiharmonic vibrations of bladed disks, ASME Journal of Engineering for Gas Turbine and Power 126 (2004) $175-183$. 
[36] M. Urabe, Galerkin's procedure for nonlinear periodic systems, Archive for Rational Mechanics and Analysis 20 (2) (1965) 120-152.

[37] R. Michalowski, Z. Mroz, Associated and non-associated sliding rules in contact friction problems, Archives of Mechanics 30 (1978) 259 - 276.

[38] A. Curnier, A theory of friction, International Journal of Solids and Structures 20 (7) (1984) 637 - 647.

[39] P. Wriggers, Computational contact mechanics, John Wiley \& Sons Ltd, 2002.

[40] P. Areias, A. P. da Costa, T. Rabczuk, F. J. M. Q. de Melo, D. D. da Costa, M. Bezzeghoud, An alternative formulation for quasi-static frictional and cohesive contact problems, Computational Mechanics 53 (4) (2014) 807-824.

[41] P. Areias, T. Rabczuk, F. J. M. Q. de Melo, J. C. de Sá, Coulomb frictional contact by explicit projection in the cone for finite displacement quasi-static problems, Computational Mechanics 55 (1) (2015) $57-72$.

[42] M. Krack, L. Salles, F. Thouverez, Vibration prediction of bladed disks coupled by friction joints, Archives of Computational Methods in Engineering (2016) 1-48.

[43] M. Krack, L. Panning, C. Siewert, Multiharmonic analysis and design of shroud friction joints of bladed disks subject to microslip, in: Proceedings of ASME IDETC/CIE, August 12-15, 2012, Chicago, IL, USA, 2012.

[44] W. J. F. Govaerts, Numerical Methods for Bifurcations of Dynamical Equilibria, Society for Industrial and Applied Mathematics Philadelphia USA, 1990.

[45] T. F. Chan, H. B. Keller, Arc-length continuation and multi-grid techniques for nonlinear elliptic eigenvalue problems, Society for Industrial and Applied Mathematics 3 (1982) 173-194. 